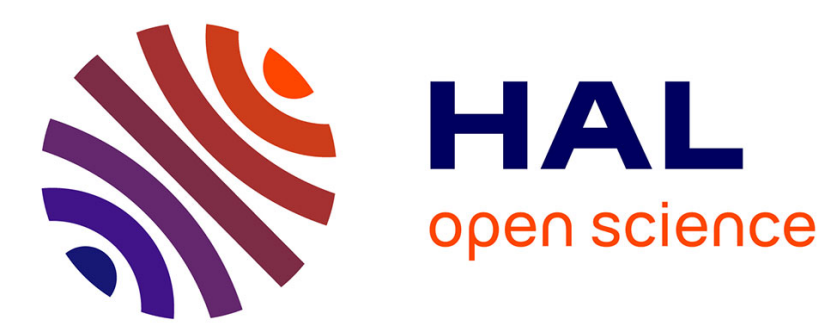

\title{
Bouncing skew Brownian motions
}

Arnaud Gloter, Miguel Martinez

\section{To cite this version:}

Arnaud Gloter, Miguel Martinez. Bouncing skew Brownian motions. 2015. hal-01223255

\section{HAL Id: hal-01223255 \\ https://hal.science/hal-01223255}

Preprint submitted on 2 Nov 2015

HAL is a multi-disciplinary open access archive for the deposit and dissemination of scientific research documents, whether they are published or not. The documents may come from teaching and research institutions in France or abroad, or from public or private research centers.
L'archive ouverte pluridisciplinaire HAL, est destinée au dépôt et à la diffusion de documents scientifiques de niveau recherche, publiés ou non, émanant des établissements d'enseignement et de recherche français ou étrangers, des laboratoires publics ou privés. 


\title{
Bouncing skew Brownian motions
}

\author{
Arnaud Gloter, Miguel Martinez
}

November 2, 2015

\begin{abstract}
We consider two skew Brownian motions, driven by the same Brownian motion, with different starting points and different skewness coefficients. In [13], the evolution of the distance between the two processes, in local time scale and up to their first hitting time is shown to satisfy a stochastic differential equation with jumps. The jumps of this S.D.E. are naturally driven by the excursion process of one of the two skew Brownian motions.

In this article, we show that the description of the distance of the two processes after this first hitting time may be studied using the self similarity induced by the previous S.D.E. More precisely, we show that the distance between the two processes in local time scale may be viewed as the unique continuous markovian self-similar extension of the process described in [13]. This permits us to compute the law of the distance of the two skew Brownian motions at any time in the local time scale, when both original skew Brownian motions start from zero. As a by product, we manage to study the markovian dependence on the skewness parameter and answer an open question formulated initially by C. Burdzy and Z.Q. Chen in [6].
\end{abstract}

MSC 2000. Primary: 60H10, Secondary: 60J55 60J65.

Key words: Skew Brownian motion; Self-similar process; Local time; Excursion process; Entrance law.

\section{Introduction}

\subsection{Presentation of the problem}

Consider $\left(B_{t}\right)_{t \geq 0}$ a standard Brownian motion on some filtered probability space $\left(\Omega, \mathcal{F},\left(\mathcal{F}_{t}\right)_{t \geq 0}, \mathbb{P}\right)$ where the filtration satisfies the usual right continuity and completeness conditions. Recall that the skew Brownian motion $X^{x, \beta}$ is defined as the solution of the stochastic differential equation with singular drift coefficient,

$$
X_{t}^{x, \beta}=x+B_{t}+\beta L_{t}^{0}\left(X^{x, \beta}\right)
$$

where $\beta \in(-1,1)$ is the skewness parameter, $x \in \mathbb{R}$, and $L_{t}^{0}\left(X^{x, \beta}\right)$ is the symmetric local time at 0 :

$$
L_{t}^{0}\left(X^{x, \beta}\right)=\lim _{\varepsilon \rightarrow 0} \frac{1}{2 \varepsilon} \int_{0}^{t} 1_{[-\varepsilon, \varepsilon]}\left(X_{s}^{x, \beta}\right) \mathrm{d} s .
$$

It is known that a strong solution of the equation (1) exists, and pathwise uniqueness holds as well (see [3], [15]). The skew Brownian motion is an example of a process partially reflected at some frontier. It finds applications 
in the fields of stochastic modeling and of numerical simulations, especially as it is deeply connected to diffusion processes with non-continuous coefficients (see [18] and references therein). The structure of the flow of a reflected, or partially reflected, Brownian motion has been the subject of several works (see e.g. [2], [5]). The long time behavior of the distance between reflected Brownian motions with different starting points has been largely studied too (see e.g. [7], [10]).

Actually, a quite intriguing fact about solutions of (1) is that they do not satisfy the usual flow property of differential equations, which prevents two solutions with different initial positions to meet in finite time. Indeed, it is shown in [2] that, when $-1<\beta_{1} \leq \beta_{2}<1$ and $x>0$, almost surely, the path $t \mapsto X_{t}^{x, \beta_{2}}$ remains above the path $t \mapsto X_{t}^{0, \beta_{1}}$, and both paths meet at an almost surely finite random time. The law of the values of the local times of these processes at the first hitting time are computed in [13]. Moreover, it is shown in [6] that in the special case $0<\frac{\beta_{2}}{1+\beta_{2}}<\beta_{1}<\beta_{2}<1$ and $x>0$ the two paths $t \mapsto X_{t}^{x, \beta_{2}}$ and $t \mapsto X_{t}^{0, \beta_{1}}$ reflect on each other. This means that, almost surely, for every $t_{0}>0$, there exists $t>0$ such that $x+\beta_{2} L_{t}^{0}\left(X^{x, \beta_{2}}\right)=\beta_{1} L_{t}^{0}\left(X^{0, \beta_{1}}\right)$ and so $X_{t}^{x, \beta_{2}}=X_{t}^{0, \beta_{1}}$ at infinitely many times (see [6] Theorem 1.4 (iii)). Since the word "reflection" is widely used in the literature in somewhat different context, we prefer to say here that the two paths "bounce" on each other.

In [13], the authors study the time dynamic of the distance between the two processes $X^{0, \beta_{1}}$ and $X^{x, \beta_{2}}$ when the skewness parameters $\beta_{1}, \beta_{2}$ are possibly different. They show that, after some random time change and to the first time they hit, the distance between the two processes is a Markov process, solution to an explicit stochastic differential equation with jumps.

This article is devoted to a detailed study of the distance between the two processes in the special case $0<\frac{\beta_{2}}{1+\beta_{2}}<\beta_{1}<\beta_{2}<1$, but after they first hit. We show that under the same random time change, this distance is the unique continuous markovian self-similar extension of the killed process described in [13]. Once this is proved, the theoretical frame provided by the theory of self-similar extensions of Markov processes permits then to compute the law of the difference process at any given deterministic time (in the new time scale). As a consequence of this study, if $\tau_{1}^{0}(B)$ denotes the inverse local time of the Brownian motion $B$ taken at time 1 , we manage to describe the inhomogeneous markovian behavior of the process $\beta \mapsto X_{\tau_{1}^{0}(B)}^{0, \beta}$ on $(0,1) \cap \mathbb{Q}$. This gives an answer to an open question first formulated by C. Burdzy and Z.Q. Chen in [6] (see [6], Open Problem $1.9)$.

\subsection{Organization of the paper}

The paper is organized as follows.

In a first section, we recall the main results obtained in our previous paper [13] that will be used in the sequel. In particular, in this first section we recall the construction of excursions of the skew Brownian motion from those of a Brownian motion and we introduce the object of study of this paper, namely the "difference process", $Z_{t}^{x, \beta_{1}, \beta_{2}}=X_{\tau_{t}\left(X^{0, \beta_{1}}\right)}^{x, \beta_{2}}$ (where $\tau_{t}\left(X^{0, \beta_{1}}\right)$ stands for the inverse local time of $\left.X^{0, \beta_{1}}\right)$, which measures the distance (looked at a proper local time scale) of two skew Brownian motions with different skewness parameters. 
In [13] this process is studied up to its first hitting time at 0 and the law of the hitting time is computed.

The second section is devoted to a first study of the "difference process" as a standard markovian reflected process. The description leads to some kind of stochastic differential equation with jumps driven by the Poisson process of excursions.

In the third section of the paper we show that the difference process is a self-similar process, extending the killed process studied in [13] to the whole time strip $[0, \infty)$ (see Proposition 2). This shows that the difference process itself admits an excursion process and a local time at 0 related through the Master formula of markovian exit systems. Based on the theory developed in [12], we identify the underlying Lévy process given via the Lamperti transform. We end this section by studying the underlying Lévy process and proving that Cramer's condition (recalled in Appendix) is satisfied for the killed difference process. This implies that there exists a self-similar extension of the killed difference process to the whole line strip $[0, \infty)$ that leaves 0 continuously (Proposition 3).

The following fourth section is then devoted to proving our first main result (Theorem 4) : namely that the extension of the killed difference process to the whole strip that leaves 0 continuously corresponds in fact to the difference process of bouncing skew Brownian motions on the whole line itself. In a subsequent section, we compute explicitely the density of the difference process $Z_{t}^{0, \beta_{1}, \beta_{2}}$ starting from the origin 0 with the help of an Itô-Dynkin formula adapted to our case. The following sections are then devoted to a brief study of the excursion process related to the difference process : we compute the entrance law of the excursion process associated to the difference process and provide a last exit decomposition. These result are obtained by direct computations.

We conclude the paper with a study of the markovian dependence of the skew Brownian motion w.r.t the skewness parameter $\beta$. The previous results allow to answer an open question formulated initially by C. Burdzy and Z.Q. Chen in [6]. In particular, we give the form of the inhomogeneous generator of the markovian process with "time" $\beta$ (see section 7.2.

\subsection{A reminder of notations and the value of constants involved in the computa- tions}

We recall here the value of constants that will be thoroughly used in this article.

1. $\beta_{1} \in(-1,1), \beta_{2} \in(-1,1)$ satisfy assumption $\mathfrak{h}: 0<\frac{\beta_{2}}{1+2 \beta_{2}}<\beta_{1}<\beta_{2}<1$.

2. $\kappa:=\frac{\left(1-\beta_{1}\right)\left(1+\beta_{2}\right)}{4 \beta_{2}} ; \gamma:=\frac{\left(1+3 \beta_{2}\right)}{2 \beta_{2}} ; \xi^{\star}:=\frac{1}{2 \beta_{1}}-\frac{1}{2 \beta_{2}} ; \theta:=\left(1-\xi^{\star}\right)$. 


\section{Bouncing skew Brownian motions}

Let $x \geq 0$. Consider the two skew Brownian motions,

$$
\begin{array}{r}
X_{t}^{x, \beta_{2}}=x+B_{t}+\beta_{2} L_{t}^{0}\left(X_{t}^{x, \beta_{2}}\right), \\
X_{t}^{0, \beta_{1}}=B_{t}+\beta_{1} L_{t}^{0}\left(X_{t}^{0, \beta_{1}}\right),
\end{array}
$$

constructed on the same probability space $\left(\Omega, \mathcal{F}, \mathbb{P}^{x}\right)$ that supports their common driving standard Brownian motion $\left(B_{t}\right)_{t \geq 0}$. When $x=0$ we will simply write $\mathbb{P}$ instead of $\mathbb{P}^{0}$.

The main issue of this paper is to study the c.a.d.l.a.g. process defined as

$$
Z_{u}^{x, \beta_{1}, \beta_{2}}=X_{\tau_{u}\left(X^{\left.0, \beta_{1}\right)}\right.}^{x, \beta_{2}}
$$

where $\tau_{u}\left(X^{0, \beta_{1}}\right)$ is the inverse of the local time of $X^{0, \beta_{1}}$, given by

$$
\tau_{u}\left(X^{0, \beta_{1}}\right)=\inf \left\{t \geq 0 \mid L_{t}^{0}\left(X^{0, \beta_{1}}\right)>u\right\}
$$

Note that, since $X_{\tau_{u}\left(X^{0, \beta_{1}}\right)}^{0, \beta_{1}}=0$, we have $Z_{u}^{x, \beta_{1}, \beta_{2}}=X_{\tau_{u}\left(X^{0, \beta_{1}}\right)}^{x, \beta_{2}}-X_{\tau_{u}\left(X^{0, \beta_{1}}\right)}^{0, \beta_{1}}$.

Since throughout this note, the parameter $\beta_{1}$ is associated to the process starting from 0 and $\beta_{2}$ to the one starting from $x$, we will, from now on, suppress the dependence upon the skewness parameter and write $X^{0}$, $X^{x}, Z^{x}$ for $X^{0, \beta_{1}}, X^{0, \beta_{2}}, Z^{x, \beta_{1}, \beta_{2}}$ when no confusion is possible.

Let us state our assumption $\mathfrak{h}$ on $\beta_{1}, \beta_{2}$ that will be used throughout this paper :

$$
\mathfrak{h}: \quad 0<\frac{\beta_{2}}{1+2 \beta_{2}}<\beta_{1}<\beta_{2}<1 .
$$

In [6] Theorem 1.4 (iii) it is proved that, almost surely, for every $t_{0}>0$, there exists $t>0$ such that $x+\beta_{2} L_{t}^{0}\left(X^{x}\right)=\beta_{1} L_{t}^{0}\left(X^{0}\right)$ and so $X_{t}^{x}=X_{t}^{0}$ at infinitely many times (see [6] Theorem 1.4 (iii)). In turn, this implies that the positive process $Z^{x}$ hits zero at infinitely many times. This justifies that, we will call below $Z^{x}$ the "difference process", which in some manner describes how both skew Brownian motion "bounce" on one another.

\subsection{Excursions of a skew Brownian motion}

Consider $X^{0, \beta}$ a skew Brownian motion starting from 0 and introduce the inverse of its local time $\tau_{u}\left(X^{0, \beta}\right)=$ $\inf \left\{t \geq 0 \mid L_{t}^{0}\left(X^{0, \beta}\right)>u\right\}$. Recall that the excursion process $\left(\mathbf{e}_{u}\right)_{u>0}$ associated to $X^{0, \beta}$ is $\mathbf{e}_{u}(r)=X_{\tau_{u-}}^{0, \beta}\left(X^{0, \beta}\right)+r$, for $r \leq \tau_{u}\left(X^{0, \beta}\right)-\tau_{u-}\left(X^{0, \beta}\right)$.

The Poisson point process $\left(\mathbf{e}_{u}\right)_{u>0}$ takes values in the space $\mathcal{C}_{0 \rightarrow 0}$ of excursions.

For $\mathbf{e} \in \mathcal{C}_{0 \rightarrow 0}$ we denote $R(\mathbf{e})$ the lifetime of the excursion and recall that by definition e does not hit zero on $(0, R(\mathbf{e}))$, and $\mathbf{e}(r)=0$ for $r \geq R(\mathbf{e})$.

If we denote $\mathbf{n}_{\beta}$ the excursion measure of the $X^{0, \beta}$, we have the formula, for any Borel subset $A$ of $\mathcal{C}_{0 \rightarrow 0}$,

$$
\mathbf{n}_{\beta}(A)=\frac{(1+\beta)}{2} \mathbf{n}_{|\mathrm{B} . \mathrm{M}|}(A)+\frac{(1-\beta)}{2} \mathbf{n}_{|\mathrm{B} . \mathrm{M}|}(-A)
$$

where $\mathbf{n}_{|\mathrm{B} . \mathrm{M}|}$ is the excursion measure for the absolute value of a Brownian motion. 


\subsection{Recalls on the difference process up to its first hit at zero}

In this paragraph, we make some recalls on known facts concerning the "distance process" derived in [13]. Up to its first hit at zero, the difference process is solution to a stochastic differential equation with jumps, driven by the excursion Poisson process of $X^{0}$.

Let us introduce $\left(\mathbf{e}_{u}\right)_{u>0}$ the excursion process associated to $X^{0, \beta_{1}}$,

$$
\mathbf{e}_{u}(r)=X_{\tau_{u-}\left(X^{0, \beta_{1}}\right)+r}^{0, \beta_{1}}, \quad \text { for } r \leq \tau_{u}\left(X^{0, \beta_{1}}\right)-\tau_{u-}\left(X^{0, \beta_{1}}\right)
$$

The Poisson point process $\left(\mathbf{e}_{u}\right)_{u>0}$ takes values in the space $\mathcal{C}_{0 \rightarrow 0}$ of excursions with finite lifetime, endowed with the usual uniform topology. Remember that $\mathbf{n}_{\beta_{1}}$ stands for the excursion measure associated to $X^{0, \beta_{1}}$.

Let us define $T^{\star}=\inf \left\{t \geq 0 \mid X_{t}^{0, \beta_{1}}=X_{t}^{0, \beta_{2}}\right\} \in[0, \infty]$ and $U^{\star}=L_{T^{\star}}^{0}\left(X_{t}^{0, \beta_{1}}\right)$. Since $X^{x, \beta_{2}}$ and $X^{0, \beta_{1}}$ are driven by the same Brownian motion, it is easy to see that they can only meet when $X^{0, \beta_{1}}=0$. As a consequence, we have

$$
U^{\star}=\inf \left\{u \geq 0 \mid Z_{u}^{x, \beta_{1}, \beta_{2}}=0\right\} \in[0, \infty], \text { and } Z^{x, \beta_{1}, \beta_{2}}>0 \text { on }\left[0, U^{\star}\right) .
$$

The description of $Z^{x}$ up to time $U^{\star}$ given in [13] is the following.

Theorem 1. (See [13] Theorem 1) Assume $x>0$ and $0<\beta_{1}, \beta_{2}<1$. Almost surely, we have for all $t<U^{\star}$,

$$
Z_{t}^{x}=x-\beta_{1} t+\sum_{0<u \leq t} \beta_{2} \ell\left(Z_{u-}^{x}, \mathbf{e}_{u}\right)
$$

where $\ell:(0, \infty) \times \mathcal{C}_{0 \rightarrow 0} \rightarrow[0, \infty)$ is a measurable map.

For $h>0$, we can describe the law of $\mathbf{e} \mapsto \ell(h, \mathbf{e})$ under $\mathbf{n}_{\beta_{1}}$ by

$$
\mathbf{n}_{\beta_{1}}\left(\ell(h, \mathbf{e}) \geq a / \beta_{2}\right)=\frac{1-\beta_{1}}{2 h}\left(1+\frac{a}{h}\right)^{-\frac{1+\beta_{2}}{2 \beta_{2}}}, \quad \forall a>0 .
$$

Corollary 1. Assume $x>0$ and $0<\beta_{1}, \beta_{2}<1$. We have for all $t<U^{\star}$,

$$
Z_{t}^{x}=x-\beta_{1} t+\int_{[0, t] \times(0, \infty)} a \mu(\mathrm{d} u, \mathrm{~d} a)
$$

where $\mu(\mathrm{d} u, \mathrm{~d} a)$ is the random jumps measure of $Z^{x, \beta_{1}, \beta_{2}}$ on $\left[0, U^{\star}\right) \times(0, \infty)$. The compensator of the measure $\mu(\mathrm{d} u, \mathrm{~d} a)$ is $\mathrm{d} u \times \nu\left(Z_{u-}^{x, \beta_{1}, \beta_{2}}, \mathrm{~d} a\right)$ with

$$
\nu(h, \mathrm{~d} a)=\frac{\kappa}{h^{2}}\left(1+\frac{a}{h}\right)^{-\gamma} 1_{\{a>0\}} \mathrm{d} a
$$

where $\kappa=\frac{\left(1-\beta_{1}\right)\left(1+\beta_{2}\right)}{4 \beta_{2}}$ and $\gamma=\frac{1+3 \beta_{2}}{2 \beta_{2}}$.

Remark 1. Theorem 1 fully details the dynamic of the "distance process" before it (possibly) reaches 0 . The "distance process" decreases with a constant negative drift, and has positive jumps. Moreover, the value of a jump at time $u$ is a function of the level $Z_{u-}^{x, \beta_{1}, \beta_{2}}$ and of the excursion $\boldsymbol{e}_{u}$. The image of the excursion measure under this function, with a fixed level $h>0$, is given by the explicit expression (7). 
Remark 2. For $0 \leq x \leq x^{\prime}$ it is possible to construct $X^{0, \beta_{1}}, X^{x, \beta_{2}}$ and $X^{x^{\prime}, \beta_{2}}$ on the same probability space. We have $X^{0, \beta_{1}} \leq X^{x, \beta_{2}} \leq X^{x^{\prime}, \beta_{2}}$ almost-surely. By time-change, we also have

$$
Z^{x, \beta_{1}, \beta_{2}} \leq Z^{x^{\prime}, \beta_{1}, \beta_{2}}
$$

Note that for any $0 \leq t \leq \frac{x}{2 \beta_{1}}$, it is easy to show that $Z_{t}^{x, \beta_{1}, \beta_{2}}$ has the same moments as the law of the jumps characterized by $\nu$. Moreover, (10) ensures that this property extends for all $t \geq 0$. In particular, we show that

$$
\mathbb{E}\left[\left(Z_{t}^{x, \beta_{1}, \beta_{2}}\right)^{\xi-1}\right]<+\infty
$$

for any $\xi \in\left[1, \frac{3}{2}+\frac{1}{2 \beta_{2}}\right)$.

Let us introduce, for $u \geq 0$, the sigma field,

$$
\mathcal{G}_{u}=\mathcal{F}_{\tau_{u}}
$$

With these notations, the process $\left(Z_{u}^{x}\right)_{u \geq 0}$ is $\left(\mathcal{G}_{u}\right)_{u \geq 0}$ adapted.

Proposition 1. (See [13] Proposition 2) Assume $x>0$ and $0>\beta_{1}<\beta_{2}<1$.

Almost surely, one has the representation for all $t<U^{\star}$

$$
L_{\tau_{t}}^{0}\left(X^{x}\right)=\sum_{0<u \leq t} \ell\left(X_{\tau_{u-}}^{x}, \mathbf{e}_{u}\right)
$$

where $\ell:(0, \infty) \times \mathcal{C}_{0 \rightarrow 0} \rightarrow[0, \infty)$ is the measurable map defined in Theorem 1 .

Moreover, we may show that almost surely if $X_{\tau_{u-}}^{x}>0$,

$$
L_{\tau_{u}}^{0}\left(X^{x}\right)-L_{\tau_{u-}}^{0}\left(X^{x}\right)=\ell\left(X_{\tau_{u-}}^{x}, \mathbf{e}_{u}\right), \quad \text { for all } u \text { with } \tau_{u}-\tau_{u-}>0
$$

Concerning the law of $U^{\star}$, it is given by the following result.

Theorem 2. (See [13] Theorem 3)

Assume $\mathfrak{h}$ and $x>0$.

Then the hitting time $T^{\star}=\inf \left\{t>0 \mid X_{t}^{0, \beta_{1}}=X_{t}^{x, \beta_{2}}\right\}$ is almost surely finite. Denote $U^{\star}=L_{T^{\star}}^{0}\left(X^{0, \beta_{1}}\right)$, then the law of $U^{\star}$ has the density

$$
p_{U^{\star}}(x, \mathrm{~d} u)=\frac{1}{\boldsymbol{b}\left(1-\xi^{\star}, \frac{1-\beta_{1}}{2 \beta_{1}}\right)} \frac{\beta_{1}}{x}\left(\frac{\beta_{1} u}{x}\right)^{\xi^{\star}-2}\left(1-\frac{x}{\beta_{1} u}\right)^{\frac{1-3 \beta_{1}}{2 \beta_{1}}} 1_{\left[\frac{x}{\beta_{1}}, \infty\right)}(u) \mathrm{d} u
$$

where $\boldsymbol{b}(a, b)=\int_{0}^{1} u^{a-1}(1-u)^{b-1} \mathrm{~d} u=\frac{\Gamma(a) \Gamma(b)}{\Gamma(a+b)}$ and $\xi^{\star}=\frac{1}{2 \beta_{1}}-\frac{1}{2 \beta_{2}}$.

Hence, $\frac{x}{\beta_{1} U^{\star}}$ is distributed as a Beta random variable $\mathcal{B}\left(1-\xi^{\star}, \frac{1-\beta_{1}}{2 \beta_{1}}\right)$.

In the next paragraph, we state the first result of this paper, which gives a first description of the "distance process" after it reaches 0 . 


\subsection{The difference process as a reflected process with jumps}

In this section, we describe the difference process as a reflected stochastic differential equation with jumps. For an account concerning reflected SDE with jumps (in the case where the jump measure is finite), we mention [14].

However, we will see that the description of $Z$ using standard methods as in [14] fails to describe precisely the behavior of the process a the boundary because of the degeneracy of the jumping measure at the boundary 0.

Theorem 3. Assume h and $x>0$.

We have for all $t \geq 0$,

$$
\begin{aligned}
Z_{t}^{x} & =x-\beta_{1} t+\beta_{2} \sum_{u<t} \mathbb{I}_{Z_{u-}^{x}>\mathrm{o}} \ell\left(Z_{u-}^{x}, \mathbf{e}_{u}\right)+\beta_{2} \sum_{u<t} \mathbb{I}_{Z_{u-}^{x}=\mathrm{o}} \int_{\tau_{u-}}^{\tau_{u}} \mathbb{I}_{\mathrm{e}_{u}(s)<0} d L_{s}^{0}\left(X^{x}\right)+\beta_{1} \int_{0}^{t} \mathbb{I}_{Z_{s}^{x}=0} d s \\
& =x-\beta_{1} t+\int_{[0, t] \times[0, \infty)} a \tilde{\mu}(\mathrm{d} u, \mathrm{~d} a)+K_{t}
\end{aligned}
$$

where

1. $\left(Z_{t}^{x}\right)_{t \geq 0}$ is an $\left(\mathcal{G}_{t}\right)$-adapted process with values in $[0, \infty)$.

2. $\tilde{\mu}(\mathrm{d} u, \mathrm{~d} a)$ is the random measure of $Z^{x}$ on $[0, \infty) \times[0, \infty)$ with compensator given by $\mathrm{d} u \times \tilde{\nu}_{u}\left(Z_{u-}^{x}, \mathrm{~d} a\right)$ with

$$
\tilde{\nu}_{u}(h, \mathrm{~d} a)=1_{\{h>0\}} \frac{\kappa}{h^{2}}\left(1+\frac{a}{h}\right)^{-\gamma} 1_{\{a>0\}} \mathrm{d} a .
$$

3. $\left(K_{t}\right)_{t \geq 0}$ is an $\left(\mathcal{G}_{t}\right)$-adapted process, null at $t=0$ and such that

$$
\int_{0}^{t} Z_{s}^{x} d K_{s}=0
$$

Remark 3. Note that in (8), the compensator of the random measure $\mu(\mathrm{d} u, \mathrm{~d} a)$ described in (9) is not welldefined for $h=0$. Contrary to the random measure $\mu(\mathrm{d} u, \mathrm{~d} a)$ in (8), the random measure $\tilde{\mu}(\mathrm{d} u, \mathrm{~d} a)$ of $(16)$ is now defined on the whole strip $[0, \infty) \times[0, \infty)$. This explains the difference between equations (8) and (16). Theorem 3 is an attempt to study the difference process in the framework provided by the theory of reflected stochastic differential equation with jumps. Unfortunately, the non-differentiable character of $\left(K_{t}\right)$ (due to the explosion of the measure describing the jumps near the boundary) does not permit to apply Itô's formula directly.

Before turning to the proof of Theorem 3, we need to clear out various preliminary results.

Lemma 1. Assume $\mathfrak{h}$ and $x>0$.

We have that

$$
\beta_{1} \int_{0}^{t} \mathbb{I}_{X_{s}^{x}=0} d L_{s}^{0}\left(X^{0}\right)=\beta_{2} \int_{0}^{t} \mathbb{I}_{X_{s}^{o}=0} d L_{s}^{0}\left(X^{x}\right) .
$$


Proof. Since $\left(X_{t}^{0}-X_{t}^{x}\right)_{t \geq 0}$ is a process with bounded variations, its local time is null. Thus, applying the Itô-Tanaka formula (for the symmetric sign function satisfying $\operatorname{sgn}(0)=0$ ), we have

$$
\begin{aligned}
X_{t}^{x} \vee X_{t}^{0} & =\left(X_{t}^{0}-X_{t}^{x}\right)^{+}+X_{t}^{x} \\
& =\int_{0}^{t}\left(\mathbb{I}_{X_{s}^{\mathrm{o}}>X_{s}^{x}}+\frac{1}{2} \mathbb{I}_{X_{s}^{\mathrm{o}}=X_{s}^{x}}\right) d\left(X_{t}^{0}-X_{t}^{x}\right)+X_{t}^{x} \\
& =x+B_{t}+\beta_{2} L_{t}^{0}\left(X^{x}\right)+\beta_{1} \int_{0}^{t} \mathbb{I}_{X_{s}^{\mathrm{o}}>X_{s}^{x}} d L_{s}^{0}\left(X_{s}^{0}\right)-\beta_{2} \int_{0}^{t} \mathbb{I}_{X_{s}^{\mathrm{o}}>X_{s}^{x}} d L_{s}^{0}\left(X_{s}^{x}\right) \\
& +\frac{1}{2}\left(\beta_{1} \int_{0}^{t} \mathbb{I}_{X_{s}^{x}=X_{s}^{\mathrm{o}}} d L_{s}^{0}\left(X^{0}\right)-\beta_{2} \int_{0}^{t} \mathbb{I}_{X_{s}^{\mathrm{o}}=X_{s}^{x}} d L_{s}^{0}\left(X^{x}\right)\right)
\end{aligned}
$$

But from the comparison principle for skew Brownian motions, we have that $\mathbb{I}_{X_{s}^{o}>X_{s}^{x}}=0$, so that

$$
\begin{aligned}
X_{t}^{x} \vee X_{t}^{0}=X_{t}^{x} & =\left(X_{t}^{0}-X_{t}^{x}\right)^{+}+X_{t}^{x} \\
& =x+B_{t}+\beta_{2} L_{t}^{0}\left(X^{x}\right)+\frac{1}{2}\left(\beta_{1} \int_{0}^{t} \mathbb{I}_{X_{s}^{x}=\mathrm{o}} d L_{s}^{0}\left(X^{0}\right)-\beta_{2} \int_{0}^{t} \mathbb{I}_{X_{s}^{\mathrm{o}=\mathrm{o}}} d L_{s}^{0}\left(X^{x}\right)\right)
\end{aligned}
$$

so that we have necessarily $(20)$.

Lemma 2. Assume $\mathfrak{h}$ and $x>0$.

$\left(Z_{t}^{x}\right)_{t \geq 0}$ is continuous at time $U^{\star}$.

Proof. This a consequence of [17] Lemma 3.2 p. 213.

Proof. (proof of Theorem 3)

Let $\mathbf{e} \in \mathcal{C}_{0 \rightarrow 0}$ and for any fixed $h>0$ consider $\mathbf{e} \mapsto \hat{X}^{h}(\mathbf{e})$ the mapping constructed in [13], which gives a possible solution of

$$
\hat{X}_{s}^{h}(\mathbf{e})=h+\mathbf{e}(s)+\beta_{2} \ell(h, \mathbf{e})
$$

with

$$
\ell(h, \mathbf{e})=\lim _{\varepsilon \searrow 0+} \frac{1}{2 \varepsilon} \int_{0}^{t} \mathbb{I}_{\hat{X}_{s}^{h}(\mathbf{e}) \in(-\varepsilon, \varepsilon)} \mathrm{d} s .
$$

It is shown in [13] that the solution of the above equation 21 is well defined for $\mathbf{n}_{\beta_{1}}$-a-e excursion $\mathbf{e} \in \mathcal{C}_{0 \rightarrow 0}$.

From (20) we deduce,

$$
\begin{aligned}
X_{t}^{x} & =x+\left(X_{t}^{0}-\beta_{1} L_{t}^{0}\left(X^{0}\right)\right)+\beta_{2} L_{t}^{0}\left(X^{x}\right) \\
& =x+\left(X_{t}^{0}-\beta_{1} L_{t}^{0}\left(X^{0}\right)\right)+\beta_{2} \int_{0}^{t} \mathbb{I}_{X_{s}^{\mathrm{o}}<\mathrm{o}} d L_{s}^{0}\left(X^{x}\right)+\beta_{2} \int_{0}^{t} \mathbb{I}_{X_{s}^{\mathrm{o}}=\mathrm{o}} d L_{s}^{0}\left(X^{x}\right) \\
& =X_{t}^{0}+x-\beta_{1} L_{t}^{0}\left(X^{0}\right)+\beta_{2} \int_{0}^{t} \mathbb{I}_{X_{s}^{\mathrm{o}<\mathrm{o}}} d L_{s}^{0}\left(X^{x}\right)+\beta_{1} \int_{0}^{t} \mathbb{I}_{X_{s}^{x}=\mathrm{o}} d L_{s}^{0}\left(X^{0}\right) .
\end{aligned}
$$

Note that the measure $\mathbb{I}_{X_{s}^{\circ}<\mathrm{o}} d L_{s}^{0}\left(X^{x}\right)$ is singular w.r.t. the measure $d L_{s}^{0}\left(X^{0}\right)$ because the ladder only increases on the set $\left\{s \geq 0: X_{s}^{0}=0\right\}$. Thus, combining the results of Proposition 1, of Lemma 2, and the equation 
$(14)$,

$$
\begin{aligned}
Z_{t}^{x} & =x-\beta_{1} t+\beta_{2} \int_{0}^{\tau_{t}} \mathbb{I}_{X_{s}^{\circ}<0} d L_{s}^{0}\left(X^{x}\right)+\beta_{1} \int_{0}^{\tau_{t}} \mathbb{I}_{X_{s}^{x}=0} d L_{s}^{0}\left(X^{0}\right) \\
& =x-\beta_{1} t+\beta_{2} \sum_{u<t} \int_{\tau_{u-}}^{\tau_{u}} \mathbb{I}_{\mathrm{e}_{u}(s)<0} d L_{s}^{0}\left(X^{x}\right)+\beta_{1} \int_{0}^{t} \mathbb{I}_{X_{\tau_{s}=0}^{x}=0} d s \\
& =x-\beta_{1} t+\beta_{2} \sum_{u<t} \mathbb{I}_{Z_{u-}^{x}>\mathrm{o}}\left(L_{\tau_{u}}^{0}\left(X^{x}\right)-L_{\tau_{u-}}^{0}\left(X^{x}\right)\right)+\beta_{2} \sum_{u<t} \mathbb{I}_{Z_{u-}^{x}=0} \int_{\tau_{u-}}^{\tau_{u}} \mathbb{I}_{\mathrm{e}_{u}(s)<0} d L_{s}^{0}\left(X^{x}\right)+\beta_{1} \int_{0}^{t} \mathbb{I}_{X_{\tau_{s}}=0} d s . \\
& =x-\beta_{1} t+\beta_{2} \sum_{u<t} \mathbb{I}_{Z_{u-}^{x}>\mathrm{o}} \ell\left(Z_{u-}^{x}, \mathbf{e}_{u}\right)+\beta_{2} \sum_{u<t} \mathbb{I}_{Z_{u-}^{x}=0} \int_{\tau_{u-}}^{\tau_{u}} \mathbb{I}_{\mathrm{e}_{u}(s)<0} d L_{s}^{0}\left(X^{x}\right)+\beta_{1} \int_{0}^{t} \mathbb{I}_{Z_{s}^{x}=0} d s .
\end{aligned}
$$

Hence, we may set

$$
K_{t}:=\beta_{2} \sum_{u<t} \mathbb{I}_{Z_{u-}^{x}=\mathrm{o}} \int_{\tau_{u-}}^{\tau_{u}} \mathbb{I}_{\mathrm{e}_{u}(s)<\mathrm{o}} d L_{s}^{0}\left(X^{x}\right)+\beta_{1} \int_{0}^{t} \mathbb{I}_{Z_{s}^{x}=0} d s .
$$

The description given at Corollary 1 gives the announced result.

\section{The difference process as a self-similar extension of the killed difference process}

In this section we show that the difference process is self-similar and extends positively the killed difference process.

\subsection{Self-similarity of the difference process}

Remember that

$$
U^{\star}:=\inf \left\{t>0: X_{\tau_{t}\left(X^{\left.0, \beta_{1}\right)}\right.}^{x, \beta_{2}}=0\right\} .
$$

Let $\left(Z_{t}^{\dagger, x}\right)_{t \leq U^{*}}=\left(X_{\tau_{t}\left(X^{0, \beta_{1}}\right)}^{x, \beta_{2}}\right)_{t \leq U^{*}}$ the process killed when it first reaches 0 . We may extend $\left(Z_{t}^{\dagger, x}\right)_{t \leq U^{*}}$ on the whole time line $[0, \infty)$ to a process - that we still note abusively $\left(Z_{t}^{\dagger, x}\right)_{t \geq 0}$ - such that 0 is a trap for $Z^{\dagger, x}$.

We have,

$$
\begin{aligned}
Z_{t}^{\dagger, x} & =x-\beta_{1} t+\sum_{0<u \leq t} \beta_{2} \ell\left(Z_{u-}^{\dagger, x}, \mathbf{e}_{u}\right), \quad t<U^{\star} \\
& =x-\beta_{1} t+\int_{[0, t] \times(0, \infty)} a \mu(\mathrm{d} u, \mathrm{~d} a), \quad t<U^{\star} .
\end{aligned}
$$

Proposition 2. Assume $\mathfrak{h}$ and $x>0$.

The process $\left(Z_{t}^{x}\right)_{t \geq 0}$ is a positive Markov 1-self-similar recurrent extension of $\left(Z_{t}^{\dagger, x}\right)_{t \geq 0}$.

Proof. First let us prove that $\left(Z_{t}^{\dagger, x}\right)_{t \geq 0}$ is a self similar process with index 1 .

Indeed, let $x>0, c>0$. Note that

$$
\int_{\mathcal{C}_{0 \rightarrow 0}} \beta_{2} \ell(h, \mathbf{e}) \mathbf{n}_{\beta_{1}}(\mathrm{de})=\int_{0}^{\infty} a \nu(h, \mathrm{~d} a)=\beta_{2} \frac{1-\beta_{1}}{1-\beta_{2}}
$$


does not depend on $h$.

Thus,

$$
\begin{aligned}
c^{-1} Z_{c t}^{\dagger, x} & =c^{-1} x-c^{-1} \beta_{1} c t+c^{-1} \sum_{0<u \leq c t} \beta_{2} \ell\left(Z_{u-}^{\dagger, x}, \mathbf{e}_{u}\right), \quad t \leq c^{-1} U^{*} \\
& =c^{-1} x+\frac{\beta_{2}-\beta_{1}}{1-\beta_{2}} t+c^{-1} \sum_{0<u \leq c t} \beta_{2} \ell\left(Z_{u-}^{\dagger, x}, \mathbf{e}_{u}\right)-c^{-1} \int_{0}^{c t} \int_{\mathcal{C}_{0 \rightarrow 0}} \beta_{2} \ell\left(Z_{u-}^{\dagger, x}, \mathbf{e}\right) \mathbf{n}_{\beta_{1}}(\mathrm{~d} \mathbf{e}) d u, \quad t \leq c^{-1} U^{\star} \\
& =c^{-1} x+\frac{\beta_{2}-\beta_{1}}{1-\beta_{2}} t+\sum_{0<v \leq t} \beta_{2} c^{-1} \ell\left(Z_{c v-}^{\dagger, x}, \mathbf{e}_{c v}\right)-\int_{0}^{t} \int_{\mathcal{C}_{0 \rightarrow 0}} \beta_{2} c^{-1} \ell\left(\bar{Z}_{c v-}^{\dagger, x}, \mathbf{e}\right) \underbrace{c \mathbf{n}_{\beta_{1}}(\mathrm{~d} \mathbf{e})}_{:=\tilde{\mathbf{n}}_{\beta_{1}}} d v, \quad t \leq c^{-1} U^{\star} .
\end{aligned}
$$

The process $Z^{c^{-1} x}:=c^{-1} Z_{c}^{\dagger, x}$ is solution of the equation :

$$
Z_{t}^{y}=y-\beta_{1} t+\sum_{0<v \leq t} \beta_{2} c^{-1} \ell\left(c Z_{v-}^{y}, \mathbf{e}_{c v}\right)
$$

for $t<\tilde{U}^{\star}:=\inf \left\{v \geq 0: Z_{v}^{y}=0\right\}$ and $y:=c^{-1} x$. The process $t \mapsto \sum_{0<v \leq t} \beta_{2} c^{-1} \ell\left(c Z_{v-}^{y}, \mathbf{e}_{c v}\right)$ is a pure jump process with compensating measure given by $d s \times \tilde{\mathbf{n}}_{\beta_{1}}(\mathrm{de})$.

Since the process $s \mapsto \mathbf{e}_{s}$ is a Poisson point process with compensating measure $d s \times \mathbf{n}_{\beta_{1}}\left(\right.$ de), so is $s \mapsto \mathbf{e}_{c s}$ with compensating measure $\mathrm{d} s \times \tilde{\mathbf{n}}_{\beta_{1}}(\mathrm{de})$ and the description of $\ell(h ; \mathbf{e})$ ensures that for $a>0$,

$$
\tilde{\mathbf{n}}_{\beta_{1}}\left(c^{-1} \ell(c h ; \mathbf{e}) \geq a\right)=c \mathbf{n}_{\beta_{1}}\left(c^{-1} \ell(c h ; \mathbf{e}) \geq a\right)=c \frac{1-\beta_{1}}{2 c h}\left(1+\frac{\beta_{2} c a}{c h}\right)^{-\frac{1+\beta_{2}}{2 \beta_{2}}}=\frac{1-\beta_{1}}{2 h}\left(1+\frac{\beta_{2} a}{h}\right)^{-\frac{1+\beta_{2}}{2 \beta_{2}}} .
$$

Consequently, we see that the 'law' of $c^{-1} \ell(c h ; \mathbf{e})$ under $\tilde{\mathbf{n}}_{\beta_{1}}$ is given by $\nu(h ; \mathrm{d} a)$. The processes $\left(Z_{t}^{\dagger, c^{-1} x}\right)_{t \geq 0}$ and $\left(c^{-1} Z_{c t}^{\dagger, x}\right)_{t \geq 0}$ share the same infinitesimal generator, start from the same point $y=c^{-1} x$, and 0 is a trap for them both : this ensures that $\left(Z_{t}^{\dagger, x}\right)_{t \geq 0}$ is a 1-self-similar process.

Second, we have that

$$
\begin{aligned}
\frac{1}{c} \frac{1}{2 \varepsilon} \int_{0}^{c^{2} t} \mathbb{I}_{[-\varepsilon, \varepsilon]}\left(X_{s}^{0}\right) d\left\langle X^{0}\right\rangle_{s} & =\frac{c}{2 \varepsilon} \int_{0}^{t} \mathbb{I}_{[-\varepsilon, \varepsilon]}\left(X_{c^{2} u}^{0}\right) d u \\
& =\frac{c}{2 \varepsilon} \int_{0}^{t} \mathbb{I}_{\left[-\frac{\varepsilon}{c}, \frac{\varepsilon}{c}\right]}\left(\frac{1}{c} X_{c^{2} u}^{0}\right) d\left\langle\frac{1}{c} X_{c^{2}}^{0}\right\rangle_{u}
\end{aligned}
$$

from which we deduce that

and

$$
\frac{1}{c} L_{c^{2} t}^{0}\left(X^{0}\right)=L_{t}^{0}\left(\frac{1}{c} X_{c^{2}}^{0}\right)
$$

$$
\left(X_{t}^{0}, L_{t}^{0}\left(X^{0}\right)\right)_{t \geq 0} \sim\left(\frac{1}{c} X_{c^{2} t}^{0}, L_{t}^{0}\left(\frac{1}{c} X_{c^{2}}^{0}\right)\right)_{t \geq 0}
$$

Moreover, since $X^{0}$ and $X^{x}$ are driven by the same Brownian motion, we have

$$
\left(X_{t}^{0}, X_{t}^{x}, L_{t}^{0}\left(X^{0}\right)\right)_{t \geq 0} \sim\left(\frac{1}{c} X_{c^{2} t}^{0}, \frac{1}{c} X_{c^{2} t}^{c x}, L_{t}^{0}\left(\frac{1}{c} X_{c^{2}}^{0}\right)\right)_{t \geq 0} .
$$

Now let $Y:=\frac{1}{c} X_{c^{2}}^{0}$. We have that

$$
\begin{aligned}
c^{2} \tau_{t}^{0}(Y) & =c^{2} \inf \left(u>0: L_{u}^{0}(Y)>t\right)=\inf \left(u>0: L_{u / c^{2}}^{0}(Y)>t\right) \\
& =\inf \left(u>0: L_{u}^{0}\left(c Y . / c^{2}\right)>c t\right)=\tau_{c t}^{0}\left(c Y . / c^{2}\right) \\
& =\tau_{c t}^{0}\left(X^{0}\right) .
\end{aligned}
$$


From the above, we deduce :

$$
\left(Z_{t}^{x}\right)_{t \geq 0}=\left(X_{\tau_{t}^{0}\left(X^{0}\right)}^{x}\right)_{t \geq 0} \sim\left(\frac{1}{c} X_{c^{2} \tau_{t}^{0}\left(\frac{1}{c} X_{c^{2}}^{0} .\right.}^{c x}\right)_{t \geq 0}=\left(\frac{1}{c} X_{\tau_{c t}^{0}\left(X^{0}\right)}^{c x}\right)_{t \geq 0}=\frac{1}{c}\left(Z_{c t}^{c x}\right)_{t \geq 0} .
$$

Consequently, $\left(Z_{t}^{x}\right)_{t \geq 0}$ is a 1 -self similar process and it is an extension of the 1-self similar process $\left(Z_{t}^{\dagger, x}\right)_{t \geq 0}$.

As mentioned in the introduction, the difference process $\left(Z_{t}^{x}\right)_{t \geq 0}$ hits zero infinitely many times, ensuring that it extends $\left(Z_{t}^{\dagger, x}\right)_{t \geq 0}$ recurrently.

Corollary 2. From [24] p. 551 or [17] p. 220, we deduce

$$
\int_{0}^{t} \mathbb{I}_{Z_{s}^{x}=0} \mathrm{~d} s=0, \quad \forall t>0, \mathbb{P}^{x}-\text { a.s. }
$$

In particular, from (16), we arrive at the description

$$
Z_{t}^{x}=x-\beta_{1} t+\beta_{2} \sum_{u<t} \mathbb{I}_{Z_{u-}^{x}>\mathrm{o}} \ell\left(Z_{u-}^{x}, \mathbf{e}_{u}\right)+\beta_{2} \sum_{u<t} \mathbb{I}_{Z_{u-}^{x}=\mathrm{o}} \int_{\tau_{u-}}^{\tau_{u}} \mathbb{I}_{\mathrm{e}_{u}(s)<\mathrm{o}} d L_{s}^{0}\left(X^{x}\right) .
$$

\subsection{Local time and excursion measure of the difference process}

Note that because 0 is a regular point there exists a local time $\left(\ell_{t}^{0}(Z)\right)$ for the difference process $\left(Z_{t}\right)$ at 0 . This is a positive continuous additive functional of the difference process $\left(Z_{t}\right)$, increasing only on the visiting set $\left\{t \geq 0: Z_{t}=0\right\}$. Such $\left(\ell_{t}^{0}(Z)\right)$ is uniquely determined up to a multiplicative constant. We normalize $\left(\ell_{t}^{0}(Z)\right)$ so that $\mathbb{E} \int_{0}^{\infty} \mathrm{e}^{-t} d \ell_{t}^{0}(Z)=1$.

Let us introduce also the inverse local time $\left(\varsigma_{t}^{0}\right)$ of $\left(\ell_{t}^{0}(Z)\right)$ and defined by

$$
\varsigma_{t}^{0}:=\inf \left(s>0: \ell_{s}^{0}(Z)>t\right), \quad t \geq 0
$$

Let $\bar{M}$ denote the closure of the zero set $\left\{t \geq 0: Z_{t}=0\right\}$ and let $\bar{G}$ denote the set of strictly positive left endpoints of the maximal intervals components of $\bar{M}^{c}$. We may associate to the excursions $\left(\mathrm{e}^{s}\right)$ of the difference process $\left(Z_{t}\right)$ away from 0 the predictable exit system $\left(\mathrm{n}, \ell^{0}(Z)\right)$, where $\mathrm{n}$ is a $\sigma$-finite measure on $\left(\Omega, \mathcal{G}^{*}\right)\left(\mathcal{G}^{*}\right.$ denotes the universal completion of $\left.\mathcal{G}^{0}\right)$ and such that if $\left(\theta_{s}\right)$ denotes the usual shift operators on $\Omega$, we have the Master formula

$$
\mathbb{E}^{x} \sum_{s \in \bar{G}} V_{s} \cdot F \circ \theta_{s}=\mathrm{n}(F) \cdot \mathbb{E}^{x} \int_{0}^{\infty} V_{s} \mathrm{~d} \ell_{s}^{0}(Z)
$$

for any predictable positive process $V$ and bounded $\mathcal{G}^{*}$ measurable functional $F$. For an arbitrary excursion e of the difference process, let us denote $R(\mathrm{e}):=\inf \left\{t>0: \mathrm{e}_{t}=0\right\}$.

Let $\left(\tilde{Z}_{t}\right)_{t \geq 0}$ denote an arbitrary self-similar extension of $\left(Z_{t}^{\dagger, x}\right)_{t \geq 0}$ with values in $[0, \infty)$ associated to an excursion measure $\tilde{n}$ and a local time $\ell^{0}(\tilde{Z})$ by a similar formula as $(25)$.

Definition 1. We say that the extension $\left(\tilde{Z}_{t}\right)_{t \geq 0}$ is a (the) 1-self-similar extension of $\left(Z_{t}^{\dagger, x}\right)_{t \geq 0}$ that leaves 0 continuously if

$$
\tilde{\mathrm{n}}\left(\tilde{Z}_{0}>0\right)=0 .
$$




\subsection{Existence and uniqueness of the positive recurrent extension that leaves zero continuously}

Proposition 3. Assume $\mathfrak{h}$ and $x>0$. There exists a unique recurrent 1 -self similar positive extension $\left(\tilde{Z}_{t}^{x}\right)_{t \geq 0}$ of $\left(Z_{t}^{\dagger, x}\right)_{t \geq 0}$ that leaves 0 continuously.

We will show later that this extension corresponds in fact to $\left(Z_{t}^{x}\right)_{t \geq 0}$ (see Section 4).

Proof. We refer to the criterion stated in Theorem 1 of [12] (see also [21]) in terms of the underlying Lévy process (the reader may look at Appendix A.3 for a brief recall concerning the underlying Lévy process and Cramer's condition).

So let us introduce the Lévy process associated with $\left(Z_{t}^{\dagger, x}\right)_{t \geq 0}$ by the Lamperti transformation (see for example [12] p. 233). For this, we consider the continuous additive functional $A^{x}$ defined by

$$
A_{t}^{x}=\int_{0}^{t} \frac{1}{Z_{s}^{\dagger, x}} d s, \quad t \geq 0
$$

and its right continuous inverse $\left(\xi_{t}^{x}\right)_{t \geq 0}$ defined by

$$
\xi_{t}^{x}=\inf \left(s>0: A_{s}^{x}>t\right), \quad t \geq 0 .
$$

We apply Ito's formula to the semi-martingale $\ln \left(Z_{t}^{\dagger, x}\right)$ for $t<U^{*}$ (recall (6)),

$$
\begin{aligned}
\ln \left(Z_{t}^{\dagger, x}\right) & =\ln (x)+\int_{0}^{t} \frac{\mathrm{d} Z_{u}^{\dagger, x}}{Z_{u-}^{\dagger, x}}+\sum_{u \leq t}\left\{\ln \left(Z_{u-}^{\dagger, x}+\Delta Z_{u}^{\dagger, x}\right)-\ln \left(Z_{u-}^{\dagger, x}\right)-\frac{\Delta Z_{u}^{\dagger, x}}{Z_{u-}^{\dagger, x}}\right\} \\
& =\ln (x)-\int_{0}^{t} \frac{\beta_{1} \mathrm{~d} u}{Z_{u}^{\dagger, x}}+\sum_{u \leq t} \ln \left(1+\frac{\Delta Z_{u}^{\dagger, x}}{Z_{u-}^{\dagger, x}}\right) .
\end{aligned}
$$

Consider the jump process $\mathcal{J}_{t}=\sum_{u \leq t} \ln \left(1+\frac{\Delta Z_{u}^{\dagger, x}}{Z_{u-}^{\dagger, x}}\right)=\sum_{u \leq t} \ln \left(1+\frac{\beta_{2} \ell\left(Z_{u-}^{\dagger, x}, \mathbf{e}_{u}\right)}{Z_{u-}^{\dagger, x}}\right)$. Its compensator can be easily computed and we have,

$$
\int_{\mathcal{C}_{0 \rightarrow 0}} \ln \left(1+\frac{\beta_{2} \ell(h, \mathbf{e})}{h}\right) \mathrm{d} \mathbf{n}_{\beta_{1}}(\mathbf{e})=\int_{0}^{\infty} \ln \left(1+\frac{a}{h}\right) \nu(h, \mathrm{~d} a)=\frac{\left(1-\beta_{1}\right) \beta_{2}}{\left(1+\beta_{2}\right) h},
$$

and hence $\widetilde{\mathcal{J}}_{t}=\mathcal{J}_{t}-\frac{\left(1-\beta_{1}\right) \beta_{2}}{\left(1+\beta_{2}\right)} \int_{0}^{t} \frac{\mathrm{d} u}{Z_{u}^{\dagger, x}}$ is a compensated jump process.

Using (27), we can write

$$
\ln \left(Z_{t}^{\dagger, x}\right)=\ln (x)+\theta \int_{0}^{t} \frac{\mathrm{d} u}{Z_{u}^{\dagger, x}}+\widetilde{\mathcal{J}}_{t}
$$

with $\theta=\frac{\left(1-\beta_{1}\right) \beta_{2}}{\left(1+\beta_{2}\right)}-\beta_{1}=\frac{\beta_{2}-\beta_{1}\left(1+2 \beta_{2}\right)}{1+\beta_{2}}<0$.

From (27) we have that

$$
H_{t}^{\ln x}:=\ln \left(Z_{\xi_{t}^{x}}^{\dagger, x}\right)=\ln (x)-\beta_{1} t+\mathcal{J}_{\xi_{t}^{x}}, \quad t \geq 0
$$

where $\mathcal{J}_{\xi_{t}^{x}}=\sum_{u \leq t} \ln \left(1+\frac{\Delta Z_{\xi_{x}}^{\dagger, x}}{Z_{\xi_{u}^{\prime}}^{\dagger, x}}\right)$ is subordinator possessing only pure jumps. 
The generator $\mathfrak{A}^{\dagger}$ of the killed process $Z^{\dagger, x}$ has domain $\mathcal{D}\left(\mathfrak{A}^{\dagger}\right)$ consisting of functions laying in the resolvent set

$$
\mathcal{D}\left(\mathfrak{A}^{\dagger}\right)=\left\{f \in \mathcal{C}^{0}([0, \infty)): \exists g \in C^{0}([0, \infty)) \text { s.t. } f(x)=\mathbb{E}^{x}\left[\int_{0}^{+\infty} \mathrm{e}^{-t} g\left(Z_{t}^{\dagger, x}\right) \mathrm{d} t\right], \forall x \geq 0\right\}
$$

and if $f \in \mathcal{D}\left(\mathfrak{A}^{\dagger}\right)$, then there exists $g \in \mathcal{C}^{0}([0, \infty))$ such that $f$ is solution of the resolvent equation

$$
f(x)-\mathfrak{A}^{\dagger} f(x)=f(x)+\beta_{1} f^{\prime}(x)-\int_{0}^{\infty}[f(x+a)-f(x)] \nu(x, \mathrm{~d} a)=g(x), \quad \forall x \geq 0 .
$$

In particular $f \in \mathcal{C}^{1}([0, \infty))$.

Letting $x$ tend to 0 in the previous equation we obtain that there exists a constant $\delta \neq 0$ such that

$$
f(0)+\delta f^{\prime}(0)=g(0)
$$

The representation of $f$ is given by

$$
f(x)=\mathbb{E}^{x}\left[\int_{0}^{+\infty} \mathrm{e}^{-t} g\left(Z_{t}^{\dagger, x}\right) \mathrm{d} t\right]=\mathbb{E}^{x}\left[\int_{0}^{U^{\star}} \mathrm{e}^{-t} g\left(Z_{t}^{\dagger, x}\right) \mathrm{d} t\right]+g(0) \mathbb{E}^{x}\left[\int_{U^{\star}}^{\infty} \mathrm{e}^{-t} \mathrm{~d} t\right]
$$

which is seen to tend to $g(0)$ as $x \searrow 0$ thanks to (15). So that by continuity of $f$ at 0 , we deduce $\delta f^{\prime}(0)=0$. Consequently,

$$
\mathcal{D}\left(\mathfrak{A}^{\dagger}\right)=\left\{f \in \mathcal{C}^{1}([0, \infty)): f^{\prime}(0)=0\right\} .
$$

Hence,

$$
\begin{aligned}
\mathfrak{A}^{\dagger} f(h) & =-\beta_{1} f^{\prime}(h)+\int_{0}^{\infty}[f(h+a)-f(h)] \nu(h, \mathrm{~d} a) \\
& =-\beta_{1} f^{\prime}(h)+\int_{0}^{\infty}[f(h+a)-f(h)] \frac{\kappa}{h^{2}}\left(1+\frac{a}{h}\right)^{-\gamma} \mathrm{d} a .
\end{aligned}
$$

for $h>0$ and $f$ an element of $\mathcal{C}^{1}[0, \infty)$ bounded on $[0, \infty)$ satisfying $f^{\prime}(0)=0$.

From the theory of time changes (see for example Lamperti [17] p. 217), we may compute the generator $\mathfrak{B}$ of $\left(H_{t}^{0}\right)_{t \geq 0}$ and we easily find that

$$
\begin{aligned}
\mathfrak{B} g(h) & =-\beta_{1} g^{\prime}(h)+\int_{0}^{\infty}[g(h+a)-g(h)] \kappa \mathrm{e}^{(\gamma-1) h} \mathrm{e}^{-(a+h)(\gamma-1)} \mathrm{d} a \\
& =-\beta_{1} g^{\prime}(h)+\int_{0}^{\infty}[g(h+a)-g(h)] \kappa \mathrm{e}^{-(\gamma-1) a} \mathrm{~d} a
\end{aligned}
$$

for $h>0$ and $g$ an element of $\mathcal{C}^{1}(0, \infty)$ bounded on $[0, \infty)$.

Consequently, the Lévy-Khintchine Formula implies

$$
\begin{aligned}
\mathbb{E}\left[\exp \left(-\lambda H_{1}^{0}\right)\right] & =\mathrm{e}^{\lambda \beta_{1}} \mathbb{E}\left[\exp \left(-\lambda \mathcal{J}_{\xi_{1}^{1}}\right)\right] \\
& =\exp \left(\lambda \beta_{1}-\kappa \int_{0}^{\infty}\left(1-\mathrm{e}^{-\lambda y}\right) \mathrm{e}^{-(\gamma-1) y} \mathrm{~d} y\right) \\
& =\exp \left(\lambda \beta_{1}-\kappa\left(\frac{1}{\gamma-1}-\frac{1}{\lambda+\gamma-1}\right)\right) .
\end{aligned}
$$


So that if $\xi^{\star}:=\frac{1}{2 \beta_{1}}-\frac{1}{2 \beta_{2}}($ as in $[13])$, we have that $\mathbb{E}\left[\exp \left(-\lambda H_{1}^{0}\right)\right]=1$ if either $\lambda=0$ or

$$
\lambda=\frac{\kappa}{\beta_{1}(\gamma-1)}-(\gamma-1)=\frac{1-\beta_{1}}{2 \beta_{1}}-\frac{1+\beta_{2}}{2 \beta_{2}}=\frac{\beta_{2}-\beta_{1}-2 \beta_{1} \beta_{2}}{2 \beta_{1} \beta_{2}}=\xi^{\star}-1,
$$

and Cramer's condition is satisfied

$$
\mathbb{E}\left[\exp \left(\left(1-\xi^{\star}\right) H_{t}^{0}\right)\right]=1 \quad \forall t>0 .
$$

Observe that for $\beta_{1}, \beta_{2}>0,\left(1-\xi^{\star}\right)>0 \Leftrightarrow \beta_{1}>\frac{\beta_{2}}{1+2 \beta_{2}}$, which is guaranteed by assumption $\mathfrak{h}$.

\section{The difference process is the positive self-similar extension of the killed distance process that leaves zero continuously}

Remember the definition (1) ; the aim of this section is to prove the following crucial result.

Theorem 4. Assume h and $x>0$.

The process $\left(Z_{t}^{x}\right)_{t \geq 0}$ is the positive Markov 1-self-similar recurrent extension of $\left(Z_{t}^{\dagger, x}\right)_{t \geq 0}$ that leaves 0 continuously.

The key of the proof relies on the preliminary results stated below.

\subsection{Existence and uniqueness for solutions of the skew Brownian equation driven by Bessel bridges}

Before getting started, let us introduce some notations.

Notations: In the next computations, we use the notation $\left(\rho(a)_{t}\right)_{t \geq 0}$ for the 3 -dimensional Bessel process starting from $a$, and $\mathbb{P}_{a}^{(3)}$ for its law. When $a=0$, we simply write $\left(\rho_{t}\right)_{t \geq 0}$.

$\mathbb{P}^{B}$ stands for the law of the standard Brownian motion.

The notation $X^{x, \beta}(\omega)$ stands for some solution of the skew Brownian motion equation driven by $\left(\omega_{t}\right)_{t \geq 0} \in$ $a+\mathbb{W}$ (where $\mathbb{W}$ is the Wiener space), namely a solution of

$$
X_{t}^{x, \beta}(\omega)=x+\omega_{t}+\beta L_{t}^{0}\left(X^{x, \beta}(\omega)\right), \quad t \geq 0
$$

where $L_{t}^{0}\left(X^{x, \beta}(\omega)\right)=\lim _{\varepsilon \searrow 0+} \frac{1}{2 \varepsilon} \int_{0}^{t} \mathbb{I}_{[-\varepsilon, \varepsilon]}\left(X^{x, \beta}(\omega)\right) \mathrm{d} s$.

The key of our proof relies on the following result

Lemma 3. Assume $-1<\beta<1$. Under $\mathbb{P}_{0}^{(3)}$, there is strong existence and uniqueness for $X^{0, \beta}(\rho)$ solution of the skew Brownian motion equation.

Moreover if $-1<\beta<1, L_{t}^{0}\left(X^{0, \beta}(\rho)\right)=0$ for any $t>0, \mathbb{P}_{0}^{(3)}$-a.s., and $X_{t}^{0, \beta}(\rho)=\rho_{t}$ for any $t>0, \mathbb{P}_{0}^{(3)}$-a.s. 
Proof. Remember that $L_{t}^{0}(\rho)=0, \mathbb{P}_{0}^{(3)}$-a.s. so that $\rho$ is itself a solution of the skew Brownian equation driven by itself. It remains to prove that it is the only solution, namely that necessarily $X^{x, \beta}(\rho)=\rho$ under $\mathbb{P}_{0}^{(3)}$.

Let $h>0$ and $a>0$ be positive parameters. Then, by Girsanov's Theorem, the process $X^{-h, \beta}(\rho(a))$ is solution of

$$
X_{t}^{-h, \beta}(\rho(a))=-h+\rho(a)_{t}+\beta L_{t}^{0}\left(X^{-h, \beta}(\rho(a))\right), \quad t \geq 0
$$

where $\left(\rho(a)_{t}\right)_{t \geq 0}$ is a standard Brownian motion starting from $a>0$ under the probability measure $\mathbb{P}_{a}$ defined by $\left.\mathbb{P}_{a}^{(3)}\right|_{\mathcal{F}_{t}}=\left.\frac{\rho(a)_{t \wedge T^{0}(\rho(a))}}{a} \mathbb{P}_{a}\right|_{\mathcal{F}_{t}}$. Because of the strong existence and uniqueness of the skew Brownian motion equation under $\mathbb{P}_{a}$, this absolute continuity relation ensures that there is strong existence and uniqueness for the solution of (35) under $\mathbb{P}_{a}^{(3)}$.

Let us now look at $X^{-h, \beta}(\rho)$, a possible solution of

$$
X_{t}^{-h, \beta}(\rho)=-h+\rho_{t}+\beta L_{t}^{0}\left(X^{-h, \beta}(\rho)\right), \quad t \geq 0 \quad \text { under } \mathbb{P}_{0}^{(3)}
$$

Using the Markov property of $\rho$, we see from the previous that equation (36) possesses a unique solution (under $\left.\mathbb{P}_{0}^{(3)}\right)$ on $\left[T^{h / 2}(\rho),+\infty\left[\right.\right.$. But the skew equation guarantees that the process $X^{-h, \beta}(\rho)$ cannot have increased its local time on the interval $\left[0, T^{h / 2}(\rho)\right]$ and $X_{t}^{-h, \beta}(\rho)=\rho_{t}$ for any $t \in\left[0, T^{h / 2}(\rho)\right]$. We thus have a solution of (36) on the whole time interval $[0, \infty)$ and this solution is likely seen to be unique.

Let $T^{1}(\rho)=\inf \left\{t>0: \rho_{t}=1\right\}$, we are going to give a lower bound on $\mathbb{P}_{0}^{(3)}\left(L_{T^{1}(\rho)}^{0}\left(X^{-h, \beta}(\rho)\right) \leq c h\right)$ where $c>0$ is some fixed constant satisfying $0<c<\frac{1}{|\beta|}$.

Note that since the local time $L^{0}\left(X^{-h, \beta}(\rho)\right)$ does not increase on $\left[0, T^{h / 2}(\rho)\right]$, the Markov property for the 3-dimensional Bessel process $\rho$ applied at $T^{h / 2}(\rho)$ gives that

$$
\mathbb{P}_{0}^{(3)}\left(L_{T^{1}(\rho)}^{0}\left(X^{-h, \beta}(\rho)\right) \leq c h\right)=\mathbb{P}_{h / 2}^{(3)}\left(L_{T^{1}(\rho(h / 2))}^{0}\left(X^{-h, \beta}(\rho(h / 2))\right) \leq c h\right) .
$$

The absolute continuity between $\mathbb{P}_{h / 2}^{(3)}$ and $\mathbb{P}_{h / 2}^{B}$ (see [22] Chap XI, exercice 1.22 p.450) for events in $\mathcal{F}_{T^{1}(\rho(h / 2))}=\mathcal{F}_{T^{1}(\rho(h / 2)) \wedge T^{0}(\rho(h / 2))}$ ensures that

$$
\mathbb{P}_{h / 2}^{(3)}\left(L_{T^{1}(\rho(h / 2))}^{0}\left(X^{-h, \beta}(\rho(h / 2))\right) \leq c h\right)=\mathbb{E}_{h / 2}^{B}\left(\mathbb{I}_{L_{T^{1}(B)}^{\circ}\left(X^{-h, \beta}(B)\right) \leq c h} \times \frac{B_{T^{1}(B) \wedge T^{0}(B)}}{h / 2}\right) .
$$

(Note that this absolute continuity relation is ensured by the fact that $\mathbb{E}_{h / 2}^{B}\left(T^{1}(B) \wedge T^{0}(B)\right)<+\infty$ and the random stopping theorem for martingales applies).

Let us evaluate $\mathbb{E}_{h / 2}^{B}\left(\mathbb{I}_{L_{\left.T^{1}(B)\right)}^{\text {o }}\left(X^{-h, \beta}(B)\right) \leq c h} \times B_{T^{1}(B) \wedge T^{0}(B)}\right)$. We have

$\mathbb{E}_{h / 2}^{B}\left(\mathbb{I}_{L_{\left.T^{1}(B)\right)}^{\mathrm{o}}\left(X^{-h, \beta}(B)\right) \leq c h} \times B_{T^{1}(B) \wedge T^{0}(B)}\right)=\mathbb{E}_{h / 2}^{B}\left(\mathbb{I}_{L_{\left.T^{1}(B)\right)}^{\mathrm{o}}\left(X^{-h, \beta}(B)\right) \leq c h} \times \mathbb{I}_{T^{1}(B) \leq T^{\circ}(B)}\right)$

$=\mathbb{E}_{h / 2}^{B}\left[\left\{\right.\right.$ there exists an excursion (necessarily positive) $\mathrm{e}_{s}$ of process $X^{-h, \beta}$ that reaches $1-h+\beta s$ while $\left.s \leq c h\right\}$

$\cap\left\{\right.$ no negative excursion $\mathrm{e}_{s}$ of process $X^{-h, \beta}$ reaches $-h+\beta s$ while $\left.\left.s \leq c h\right\}\right]$

$=\mathbb{P}_{h / 2}^{B}\left[\left\{\right.\right.$ there exists an excursion (necessarily positive) $\mathrm{e}_{s}$ of process $X^{-h, \beta}$ that reaches $1-h+\beta s$ while $\left.\left.s \leq c h\right\}\right]$ $\times \mathbb{P}_{h / 2}^{B}\left[\left\{\right.\right.$ no (necessarily negative) excursion $\mathrm{e}_{s}$ of process $X^{-h, \beta}$ reaches $-h+\beta s$ while $\left.\left.s \leq c h\right\}\right]$, 
where the last equality comes from the independence of the processes of positive and negative excursions and because $c \in\left(0, \frac{1}{|\beta|}\right)$.

We compute $\mathbb{P}_{h / 2}^{B}\left[\left\{\right.\right.$ there exists an excursion (necessarily positive) $\mathrm{e}_{s}$ of process $X^{-h, \beta}$ that reaches $1-h+\beta s$ while $\left.\left.s \leq c h\right\}\right]$ $=1-\left(1+\frac{\beta c h}{1+h}\right)^{-\frac{1+\beta}{2 \beta}}$

and

$$
\begin{aligned}
& \mathbb{P}_{h / 2}^{B}\left[\left\{\text { no negative excursion } \mathrm{e}_{s} \text { of process } X^{-h, \beta} \text { reaches }-h+\beta s \text { while } s \leq c h\right\}\right] \\
& =(1-c \beta)^{\frac{1-\beta}{2 \beta}}
\end{aligned}
$$

Finally, from (37) and (38) we deduce

$$
\mathbb{P}_{0}^{(3)}\left(L_{T^{1}(\rho)}^{0}\left(X^{-h, \beta}(\rho)\right) \leq c h\right)=\frac{2}{h}\left(1-\left(1+\frac{\beta c h}{1+h}\right)^{-\frac{1+\beta}{2 \beta}}\right)(1-c \beta)^{\frac{1-\beta}{2 \beta}} .
$$

In particular, we see that there exists $p>0$ satisfying

$$
\mathbb{P}_{0}^{(3)}\left(L_{T^{1}(\rho)}^{0}\left(X^{-h, \beta}(\rho)\right) \leq c h\right) \geq p>0
$$

uniformly for any $h>0$ sufficiently small.

We are now ready to show uniqueness for solutions $X^{0, \beta}(\rho)$ for $\beta \in(-1,1)$ (case $h=0$ ). The difficult case is when $\beta \in(-1,0)$, so we now assume that $\beta \in(-1,0)$. Let us denote

$$
X_{t}^{0-, \beta}(\rho)=\sup _{x<0, x \in \mathbb{Q}} X_{t}^{x, \beta}(\rho) ; L_{t}^{0}\left(X^{0-, \beta}(\rho)\right)=\sup _{x<0, x \in \mathbb{Q}} L_{t}^{0}\left(X^{x, \beta}(\rho)\right) .
$$

Suppose that we have proven that

$$
\mathbb{P}_{0}^{(3)}\left(\bigcup_{s \in\left[0, T^{1}(\rho)\right)}\left\{L_{t}^{0}\left(X^{0-, \beta}(\rho)\right)=0, \forall t \in[0, s]\right\}\right)=1 .
$$

Then, remember that there exists simultaneous solutions of (36) when $h \in \mathbb{Q}^{+}$. The comparison theorem for solutions of the skew Brownian motion equation ensures that a.s. for any $t \geq 0$, the family of r.v. $\left\{X_{t}^{-h, \beta} h \in\right.$ $\left.\mathbb{Q}^{+}\right\}$is a.s. increasing w.r.t. $-h$, so that

$$
L_{t}^{0}\left(X^{-h, \beta}(\rho)\right) \geq \frac{h}{\beta}+L_{t}^{0}\left(X^{0, \beta}(\rho)\right)
$$

for any fixed $h>0$. Letting $h \in \mathbb{Q}^{+}$tend to 0 in the previous inequality gives

$$
L_{t}^{0}\left(X^{0-, \beta}(\rho)\right) \geq L_{t}^{0}\left(X^{0, \beta}(\rho)\right)
$$

Consequently, if (41) is proved, then there exists a (possibly random) $s>0$ such that $L_{t}^{0}\left(X^{0, \beta}(\rho)\right)=0, \forall t \in[0, s]$ $\mathbb{P}_{0}^{(3)}$-a.s. But if this is the case, then $X_{s}^{0, \beta}(\rho)=\rho_{s}>0$, and since $\rho$ does not hit 0 after time $s>0, X^{0, \beta}(\rho)$ 
will never hit 0 either after $s>0$ because it satisfies the skew Brownian equation driven by $\rho$. This proves that $X^{0, \beta}(\rho)$ cannot increase its local time and the result of the lemma follows directly.

It remains to prove (41).

From (40), for any $\varepsilon>0$

$$
\mathbb{P}_{0}^{(3)}\left(\bigcup_{h \in(0, \varepsilon), h \in \mathbb{Q}^{+}}\left\{L_{T^{1}(\rho)}^{0}\left(X^{-h, \beta}(\rho)\right) \leq c h\right\}\right) \geq p
$$

and Fatou's lemma implies

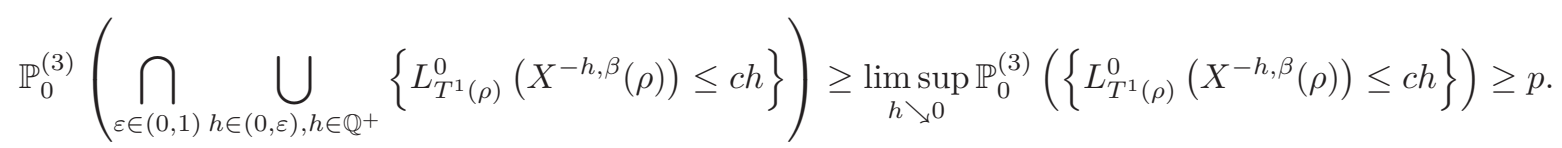

So that

$$
\mathbb{P}_{0}^{(3)}\left(\limsup _{h \searrow 0}\left\{L_{T^{1}(\rho)}^{0}\left(X^{-h, \beta}(\rho)\right) \leq c h\right\}\right)=\mathbb{P}_{0}^{(3)}\left(L_{T^{1}(\rho)}^{0}\left(X^{0-, \beta}(\rho)\right)=0\right) \geq p .
$$

In particular, since $s \mapsto L_{s}^{0}\left(X^{0-, \beta}(\rho)\right)$ is a.s. increasing and $T^{1}(\rho)>0$ a.s., we have

$$
\mathbb{P}_{0}^{(3)}\left(\cup_{s \in\left[0, T^{1}(\rho)\right)}\left\{L_{t}^{0}\left(X^{0-, \beta}(\rho)\right)=0, \forall t \in[0, s]\right\}\right) \geq \mathbb{P}_{0}^{(3)}\left(\left\{L_{T^{1}(\rho)}^{0}\left(X^{0-, \beta}(\rho)\right)=0\right\}\right) \geq p .
$$

Note that the event $\cup_{s \in\left[0, T^{1}(\rho)\right)}\left\{L_{t}^{0}\left(X^{0-, \beta}(\rho)\right)=0, \forall t \in[0, s]\right\}$ belongs to the germ $\sigma$-field $\mathcal{F}_{0+}=\cap_{s>0} \sigma\left(\rho_{t}, 0<t<s\right)$. So, applying Blumenthal's zero-one law and since $p>0$, we necessarily conclude that

$$
\mathbb{P}_{0}^{(3)}\left(\cup_{s \in\left[0, T^{1}(\rho)\right)}\left\{L_{t}^{0}\left(X^{0-, \beta}(\rho)\right)=0, \forall t \in[0, s]\right\}\right)=1,
$$

which is exactly (41) and the result is proved.

Let $\mathbb{P}_{0,0}^{(3), r}$ the law of the Bessel bridge of dimension 3 over $[0, r]$. From the previous lemma, we readily deduce the following corollary.

Corollary 3. Assume $-1<\beta<1$. Under $\mathbb{P}_{0,0}^{(3), r}$, there is strong existence and uniqueness for $X^{0, \beta}(\omega)$ solution of the skew Brownian motion equation.

Moreover, $L_{t}^{0}\left(X^{0, \beta}(\omega)\right)=0$ for any $0 \leq t \leq r, \mathbb{P}_{0,0}^{(3), r}$-a.s., and $X_{t}^{0, \beta}(\omega)=\omega_{t}$ for any $0 \leq t \leq r, \mathbb{P}_{0,0}^{(3), r}-a . s$. .

Proof. We give the main idea of the proof, leaving the details to the reader.

Using the result of Lemma 3 and the absolute continuity relationship between $\mathbb{P}_{0,0}^{(3), r}$ and $\mathbb{P}_{0}^{(3)}$ (see for example [22] Exercice 3.11 p.468) we get that under $\mathbb{P}_{0,0}^{(3), r}$, there is existence and uniqueness for $X^{0, \beta}(\omega)$ solution of the skew Brownian motion equation on any time interval $0 \leq t<r-\varepsilon(0<\varepsilon<r)$. Moreover, we have $L_{t}^{0}\left(X^{0, \beta}(\omega)\right)=0$ for any $0 \leq t<r-\varepsilon(0<\varepsilon<r)$.

But if this is the case, $X_{r-\varepsilon}^{0, \beta}(\omega)=\omega_{r-\varepsilon}>0$, and since under $\mathbb{P}_{0,0}^{(3), r}$ the trajectory $\omega$ does not hit 0 before time $r>0$ a.s., $X^{0, \beta}(\omega)$ will never hit 0 either before $r$ (because it satisfies the skew Brownian equation driven by $\omega)$. This proves that $\mathbb{P}_{0,0}^{(3), r}$-a.s. $X^{0, \beta}$ cannot increase its local time before the end time $r>0$ and the result of the corollary follows. 


\subsection{Conclusion : proof of Theorem 4}

Proof. We will now use the master formula (25)

$$
\mathbb{E}^{x} \sum_{s \in \bar{G}} V_{s} \cdot F \circ \theta_{s}=\mathrm{n}(F) \cdot \mathbb{E}^{x} \int_{0}^{\infty} V_{s} \mathrm{~d} \ell_{s}^{0}(Z)
$$

for any predictable positive process $V$ and bounded $\mathcal{G}^{*}$ measurable functional $F$.

Let us choose $T>0$ satisfying $\mathbb{E}^{x} \ell_{T}^{0}(Z)>0$ (remember the normalization $\mathbb{E}^{0} \int_{0}^{\infty} \mathrm{e}^{-s} \ell_{s}^{0}(Z) \mathrm{d} s=1$ so that it is easily seen that such $T$ exists) and set $F=\mathbb{I}_{\lim _{u} \searrow_{0}} Z_{u}>0=\mathbb{I}_{Z_{\mathrm{o}}>\mathrm{o}}=\mathbb{I}_{\lim _{u} \searrow_{\mathrm{o}} X_{\tau_{u}^{\mathrm{o}}}^{x}>\mathrm{o}}$ and $V_{s} \equiv \mathbb{I}_{[\mathrm{o}, T]}(s)$.

Fact 1 : first note that $\forall t \in \bar{G}, Z_{t-}^{x}=0$.

Indeed, by definition of $\bar{G}$, if $t$ lies in $\bar{G}$, there exists a sequence of times $\left(u_{n}\right)$ such that $u_{n} \uparrow t$ with $Z_{u_{n}}^{x}=0$. Since $Z$ has a.s. rcll trajectories, we have $0=\lim _{u_{n} \uparrow t} Z_{u_{n}}^{x}=Z_{t-}^{x}$.

Fact 2 : second, note that if $t \in \bar{G}$ and $Z_{t}^{x}>0$, then necessarily $\tau_{t-}^{0} \neq \tau_{t}^{0}$.

Indeed, by the preceding (fact 1), $Z_{\tau_{t-}^{0}}^{x}=X_{\tau_{t-}^{0}}^{x, \beta_{2}}=0$. But if $\tau_{t}^{0}=\tau_{t-}^{0}$, then so would we have $Z_{t}^{x}=X_{\tau_{t}^{0}}^{x, \beta_{2}}=0$, violating our hypothesis $Z_{t}^{x}>0$.

Let us denote $\mathcal{Z}=\left\{s \in \mathbb{R}^{+}: X_{s}^{0, \beta_{1}}=0\right\}$ the zero-set of $X^{0, \beta_{1}}$ and $\mathcal{Z}_{g}$ the left-hand points of the maximal intervals in $\mathcal{Z}^{c}$.

From fact 1 and fact 2, we deduce

$$
\begin{aligned}
& 0 \leq \mathbb{E}^{x} \sum_{s \in \bar{G}} V_{s} . F \circ \theta_{s}=\mathbb{E}^{x} \sum_{s \in \bar{G}, \tau_{s-}^{0} \neq \tau_{s}^{0}} V_{s} \cdot \mathbb{I}_{X_{\tau^{0}-}^{x, \beta_{2}=0}} \cdot F \circ \theta_{s} \\
& \leq \mathbb{E}^{x} \sum_{\left\{s \mid \tau_{s-}^{0} \neq \tau_{s}^{0}\right\}} V_{s} \cdot \mathbb{I}_{X_{\tau_{s-}^{0}+R\left(\mathrm{e}_{s}\right)}^{x, \beta_{2}}}>\mathrm{o}, X_{\tau_{s-}^{\mathrm{o}}}^{x, \beta_{2}}=0 \\
& \leq \mathbb{E}^{x} \sum_{s \in \mathcal{Z}_{g}} V_{s} \cdot H \circ \theta_{s}
\end{aligned}
$$

with $H=\mathbb{I}_{L_{R(\mathrm{e})}^{\mathrm{o}}\left(X^{\mathrm{o}, \beta_{2}}(\mathrm{e})\right)>0}$. Now applying the Master formula for the skew Brownian motion (with parameter $\left.\beta_{1}\right)$ and the result of Corollary 3 gives

$$
\mathbb{E}^{x} \sum_{s \in \mathcal{Z}_{g}} V_{s} . H \circ \theta_{s}=\mathbf{n}_{\beta_{1}}\left(L_{R(\mathrm{e})}^{0}\left(X^{\left.0, \beta_{2}\right)(\mathrm{e})}>0\right) \mathbb{E}^{x} L_{T}^{0}\left(X^{0, \beta_{1}}\right)=0\right.
$$

where we used

$$
\mathbf{n}_{\beta_{1}}^{+}\left(L_{R(\mathrm{e})}^{0}\left(X^{0, \beta_{2}}(\mathrm{e})\right)>0\right)=\int_{0}^{\infty} \frac{1}{2 \sqrt{2 \pi r^{3}}} \mathbb{P}_{0,0}^{(3), r}\left(L_{r}^{0}\left(X^{0, \beta_{2}}(\omega)\right)>0\right) \mathrm{d} r=0
$$

thanks to the result of Corollary 3 . Of course the same holds for $\mathbf{n}_{\beta_{1}}^{-}\left(L_{R(\mathrm{e})}^{0}\left(X^{0, \beta_{2}}(\mathrm{e})\right)>0\right)$.

Coming back to (43), this implies

$$
0=\mathbb{E}^{x} \sum_{s \in \bar{G} \cap[0, T]} F \circ \theta_{s}=\mathrm{n}(F) \cdot \mathbb{E}^{x} \ell_{T}^{0}(Z)
$$

and $\mathrm{n}(F)=\mathrm{n}\left(Z_{0}>0\right)=0$. The theorem is thus proved. 
Corollary 4. The result of Theorem 4 ensures finally that

$$
\beta_{2} \sum_{u<t} \mathbb{I}_{Z_{u-}^{x}=0} \int_{\tau_{u-}}^{\tau_{u}} \mathbb{I}_{\mathrm{e}_{u}(s)<0} d L_{s}^{0}\left(X^{x}\right)=0
$$

almost everywhere under $\mathbf{n}_{\beta_{1}}$.

From (16) and the result of Corollary 2 eq-(24), we finally arrive at the description

$$
Z_{t}^{x}=x-\beta_{1} t+\beta_{2} \sum_{u<t} \mathbb{I}_{Z_{u-}^{x}>\mathrm{o}} \ell\left(Z_{u-}^{x}, \mathbf{e}_{u}\right) \quad t \geq 0
$$

and $K \equiv 0$.

From this last equation (45), we see that the description of $Z$ using the usual theory of reflected jumping processes fails to describe the trajectories at the reflecting boundary 0 .

\section{The law of the difference process starting at zero}

Let us define the family of probability measures $\left(\mathbb{P}^{x} ; x>0\right)$ on the Skorokhod space $\mathcal{D}$ by

$$
\mathbb{P}^{x}(A)=\mathbb{P}\left(\left(t \mapsto Z_{t}^{x}\right) \in A\right)
$$

As a consequence of the previous study, we know that the process $\left(Z^{x}, \mathbb{P}^{x} ; x>0\right)$ is a self-similar Markov process. The Markov property implies that the process $\left(Z_{U^{\star}+t}^{x}, t \geq 0\right)$ is independent of the process $\left(Z_{U^{\star}+t}^{x}, t \leq U^{\star}\right)$ and its law does not depend on $x$. Moreover, the scaling property implies that $\lim _{x \rightarrow 0} U^{\star}=0$ a.s., hence, this shows that the family of measures $\left(\mathbb{P}^{x} ; x>0\right)$ converges weakly, as $x$ goes to 0 , towards the law of the process $\left(Z_{U^{\star}+t}^{x}, t \geq 0\right)$. Let us mention that in this case Rivero [21] gives a construction of an entrance law for the process $\left(Z_{U^{\star}+t}^{x}, t \geq 0\right)$ in terms of exponential functionals of the underlying Levy process. We will denote by $\left(Z^{0}, \mathbb{P}^{0}\right)$ a process whose law $\mathbb{P}^{0}$ is that of the process $\left(Z_{U^{\star}+t}^{x}, t \geq 0\right)$. The process $\left(Z^{0}, \mathbb{P}^{0}\right)$ will be called a "difference process starting from zero". By construction, the processes $\left(Z^{0}, \mathbb{P}^{0}\right)$ and $\left(Z^{x}, \mathbb{P}^{x}\right)$ share a common infinitesimal generator.

\subsection{An Itô-Dynkin formula for flat functions near zero}

The aim of this paragraph is to prove the following proposition.

Proposition 4. Assume that $f: \mathbb{R}^{+} \rightarrow \mathbb{R}$ is a $\mathcal{C}^{1}$ function such that there exists a neighborhood $V \subset[0,1)$ of $\{0\}$ and some $\delta>0$ such that $\left|f^{\prime}(y)\right| \leq C y^{\delta}$ for any $y \in V$. Then, we have the following Itô-Dynkin formula for $f$ :

$$
\mathbb{E}\left(f\left(Z_{t}^{x}\right)-f(x)\right)=-\beta_{1} \int_{0}^{t} \mathbb{E}\left(f^{\prime}\left(Z_{s}^{x}\right)\right) d s+\int_{0}^{t} d s \int_{0}^{\infty} \mathbb{E}\left[\left(f\left(Z_{s-}^{x}+a\right)-f\left(Z_{s-}^{x}\right)\right) 1_{Z_{s-}^{x}>0} \tilde{\nu}\left(Z_{s-}^{x}, d a\right)\right]
$$

Before proving Proposition 4, we need the following Lemma : 
Lemma 4. We have that

$$
\mathbb{P}\left(Z_{t}^{x}=0\right)=0
$$

for any $t \geq 0$, for any $x>0$.

Proof. From (23) and taking expectations $\mathbb{E}$ that the equality (48) is satisfied for almost every $t>0$ outside a negligible $\mathcal{N}_{x}$ set. By the absurd suppose there exists $t_{0}>0$ and $x>0$ with $\mathbb{P}\left(Z_{t_{0}}^{x}=0\right)>\delta\left(\right.$ i.e. $\left.t_{0} \in \mathcal{N}_{x}\right)$. There exists $a>1, t_{1}=a t_{0}>>t_{0}$ with $\mathbb{P}\left(Z_{t_{1}}^{x}=0\right)=0$. Then, by the comparison principle for time changed skew Brownian motions, we have

$$
0=\mathbb{P}\left(Z_{t_{1}}^{x}=0\right)=\mathbb{P}\left(Z_{a t_{0}}^{x}=0\right)=\mathbb{P}\left(Z_{t_{0}}^{x / a}=0\right) \geq \mathbb{P}\left(Z_{t_{0}}^{x}=0\right)>\delta
$$

yielding the contradiction.

Proof. (of Proposition 4)

Let $\varepsilon>0$ be fixed. Set $\tau_{\varepsilon}^{0}=\tau_{2 \varepsilon}^{0}=0$,

$\tau_{\varepsilon}^{k+1}=\inf \left(s \geq \tau_{2 \varepsilon}^{k}: Z_{s}^{x}=\varepsilon\right)$ for any integer $k \geq 0 ; \tau_{2 \varepsilon}^{k}=\inf \left(s \geq \tau_{\varepsilon}^{k}: Z_{s}^{x} \geq 2 \varepsilon\right)$ for any integer $k \geq 1$.

For convenience, we introduce the notation $[\triangle f](y, a)=f(a+y)-f(y)$ for $y \geq 0, a \geq 0$.

Using the Markovian nature of $\left(Z_{t}^{x}\right)$ and using repeatedly Itô's formula on the intervals $\left[\tau_{2 \varepsilon}^{k}, \tau_{\varepsilon}^{k+1}\right]$ (allowed by the fact that on these intervals, the generator of $\left(Z_{t}^{x}\right)_{t \geq 0}$ is completely known), for $x>2 \varepsilon$ we may write

$$
\begin{aligned}
& f\left(Z_{t}^{x}\right)-f(x) \\
& =1_{Z_{t}^{x} \geq 2 \varepsilon} \sum_{k=0}^{\infty}\left\{\left[\left(f\left(Z_{\tau_{\varepsilon}^{k+1}}^{x}\right)-f\left(Z_{\tau_{2 \varepsilon}^{k}}^{x}\right)\right)+\left(f\left(Z_{\tau_{2 \varepsilon}^{k}}^{x}\right)-f\left(Z_{\tau_{\varepsilon}^{k}}^{x}\right)\right)\right] 1_{\tau_{2 \varepsilon}^{k} \leq t}-\left(f\left(Z_{\tau_{\varepsilon}^{k+1}}^{x}\right)-f\left(Z_{t}^{x}\right)\right) 1_{\tau_{2 \varepsilon}^{k} \leq t \leq \tau_{\varepsilon}^{k+1}}\right\} \\
& \quad+1_{Z_{t}^{x}<2 \varepsilon}\left(f\left(Z_{t}^{x}\right)-f(x)\right) \\
& =1_{Z_{t}^{x} \geq 2 \varepsilon} \sum_{k=0}^{\infty}\left[\left(M_{\tau_{\varepsilon}^{k+1}}^{f}-M_{\tau_{2 \varepsilon}^{k}}^{f}\right)-\beta_{1} \int_{\tau_{2 \varepsilon}^{k}}^{\tau_{\varepsilon}^{k+1}} f^{\prime}\left(Z_{s}^{x}\right) d s+\int_{\left(\tau_{2 \varepsilon}^{k}, \tau_{\varepsilon}^{k+1}\right]} d s \int_{0}^{\infty}[\Delta f]\left(Z_{s-}^{x}, a\right) \tilde{\nu}\left(Z_{s-}^{x}, d a\right)\right] 1_{\tau_{2 \varepsilon}^{k} \leq t} \\
& \quad-1_{Z_{t}^{x} \geq 2 \varepsilon} \sum_{k=0}^{\infty}\left[\left(M_{\tau_{\varepsilon}^{k+1}}^{f}-M_{t}^{f}\right)-\beta_{1} \int_{t}^{\tau_{\varepsilon}^{k+1}} f^{\prime}\left(Z_{s}^{x}\right) d s+\int_{t}^{\tau_{\varepsilon}^{k+1}} d s \int_{0}^{\infty}[\triangle f]\left(Z_{s-}^{x}, a\right) \tilde{\nu}\left(Z_{s-}^{x}, d a\right)\right] 1_{\tau_{2 \varepsilon}^{k} \leq t \leq \tau_{\varepsilon}^{k+1}} \\
& \quad+1_{Z_{t}^{x} \geq 2 \varepsilon} \sum_{k=0}^{\infty}\left(f\left(Z_{\tau_{2 \varepsilon}^{k}}^{x}\right)-f\left(Z_{\tau_{\varepsilon}^{k}}^{x}\right)\right) 1_{\tau_{2 \varepsilon}^{k} \leq t}+1_{Z_{t}^{x}<2 \varepsilon}\left(f\left(Z_{t}^{x}\right)-f(x)\right) .
\end{aligned}
$$

So that, by adding and subtracting the missing bounded variation terms on the intervals $\left(\tau_{\varepsilon}^{k}, \tau_{2 \varepsilon}^{k}\right]$ in order to complete the integrals, we have

$$
\begin{aligned}
& f\left(Z_{t}^{x}\right)-f(x) \\
& =1_{Z_{t}^{x} \geq 2 \varepsilon}\left(\sum_{k=0}^{\infty}\left(M_{\tau_{\varepsilon}^{k+1}}^{f}-M_{\tau_{2 \varepsilon}^{k}}^{f}\right)-\beta_{1} \int_{0}^{t} f^{\prime}\left(Z_{s}^{x}\right) d s+\int_{0}^{t} d s \int_{0}^{\infty}[\Delta f]\left(Z_{s-}^{x}, a\right) 1_{Z_{s-}^{x}>0} \tilde{\nu}\left(Z_{s-}^{x}, d a\right)\right) \\
& \quad-\sum_{k=0}^{\infty}\left[-\beta_{1} \int_{\tau_{\varepsilon}^{k}}^{\tau_{2 \varepsilon}^{k}} f^{\prime}\left(Z_{s}^{x}\right) d s+\int_{\left(\tau_{\varepsilon}^{k}, \tau_{2 \varepsilon}^{k}\right]} d s \int_{0}^{\infty}[\triangle f]\left(Z_{s-}^{x}, a\right) 1_{Z_{s-}^{x}>0} \tilde{\nu}\left(Z_{s-}^{x}, d a\right)\right] 1_{\tau_{2 \varepsilon}^{k} \leq t} \\
& \quad-1_{Z_{t}^{x} \geq 2 \varepsilon} \sum_{k=0}^{\infty}\left(M_{\tau_{\varepsilon}^{k+1}}^{f}-M_{t}^{f}\right) 1_{\tau_{2 \varepsilon}^{k} \leq t \leq \tau_{\varepsilon}^{k+1}}+1_{Z_{t}^{x} \geq 2 \varepsilon} \sum_{k=0}^{\infty}\left(f\left(Z_{\tau_{2 \varepsilon}^{k}}^{x}\right)-f\left(Z_{\tau_{\varepsilon}^{k}}^{x}\right)\right) 1_{\tau_{2 \varepsilon}^{k} \leq t}+1_{Z_{t}^{x}<2 \varepsilon}\left(f\left(Z_{t}^{x}\right)-f(x)\right) .
\end{aligned}
$$


We may complete the martingale increments too and we may write (with a slight abuse of notation)

$$
\begin{aligned}
& f\left(Z_{t}^{x}\right)-f(x) \\
& =1_{Z_{t}^{x} \geq 2 \varepsilon}\left(M_{t}^{f, \varepsilon}-M_{0}^{f, \varepsilon}-\beta_{1} \int_{0}^{t} f^{\prime}\left(Z_{s}^{x}\right) d s+\int_{0}^{t} d s \int_{0}^{\infty}[\triangle f]\left(Z_{s-}^{x}, a\right) 1_{Z_{s-}^{x}>0} \tilde{\nu}\left(Z_{s-}^{x}, d a\right)\right) \\
& \quad+1_{Z_{t}^{x} \geq 2 \varepsilon} \sum_{k=0}^{\infty}\left[\left(f\left(Z_{\tau_{2 \varepsilon}^{k}}^{x}\right)-f\left(Z_{\tau_{2 \varepsilon}^{k}-}^{x}\right)\right)\right] 1_{\tau_{2 \varepsilon}^{k} \leq t} \\
& \quad-\sum_{k=0}^{\infty}\left[-\beta_{1} \int_{\tau_{\varepsilon}^{k}}^{\tau_{2 \varepsilon}^{k}} f^{\prime}\left(Z_{s}^{x}\right) d s+\int_{\left(\tau_{\varepsilon}^{k}, \tau_{2 \varepsilon}^{k}\right]} d s \int_{0}^{\infty}[\Delta f]\left(Z_{s-}^{x}, a\right) 1_{Z_{s-}^{x}>0} \tilde{\nu}\left(Z_{s-}^{x}, d a\right)\right] 1_{\tau_{2 \varepsilon}^{k} \leq t} \\
& \quad+1_{Z_{t}^{x} \geq 2 \varepsilon} \sum_{k=0}^{\infty}\left(f\left(Z_{\tau_{2 \varepsilon}^{k}-}^{x}\right)-f\left(Z_{\left.\tau_{\varepsilon}^{k}\right)}^{x}\right)\right) 1_{\tau_{2 \varepsilon}^{k} \leq t}+1_{Z_{t}^{x}<2 \varepsilon}\left(f\left(Z_{t}^{x}\right)-f(x)\right)
\end{aligned}
$$

where $\left(M_{t}^{f, \varepsilon}\right)$ is a martingale which is constant on the intervals of type $\left(\tau_{\varepsilon}^{k}, \tau_{2 \varepsilon}^{k}\right]$.

So that

$$
\begin{aligned}
& \mid\left(f\left(Z_{t}^{x}\right)-f(x)\right)-\left(M_{t}^{f, \varepsilon}-M_{0}^{f, \varepsilon}\right) \\
& \quad-\beta_{1} \int_{0}^{t} f^{\prime}\left(Z_{s}^{x}\right) d s+\int_{0}^{t} d s \int_{0}^{\infty}[\triangle f]\left(Z_{s-}^{x}, a\right) 1_{Z_{s-}^{x}>0} \tilde{\nu}\left(Z_{s-}^{x}, d a\right) \mid \\
& \leq 1_{Z_{t}^{x} \geq 2 \varepsilon} \sum_{k=0}^{\infty}\left|f\left(Z_{\tau_{2 \varepsilon}^{k}}^{x}\right)-f\left(Z_{\tau_{2 \varepsilon}^{k}-}^{x}\right)\right| 1_{\tau_{2 \varepsilon}^{k} \leq t} \\
& \quad+\sum_{k=0}^{\infty} 1_{\tau_{2 \varepsilon}^{k} \leq t} \int_{\left(\tau_{\varepsilon}^{k}, \tau_{2 \varepsilon}^{k}-\right]} d s \sup _{z \in[0,2 \varepsilon)}\left|f^{\prime}(z)\right| \int_{0}^{\infty} a 1_{Z_{s-}^{x}>0} \tilde{\nu}\left(Z_{s-}^{x}, d a\right) 1_{\tau_{2 \varepsilon}^{k} \leq t} \\
& \quad+\left|\beta_{1}\right| t \sup _{z \in[0,2 \varepsilon)}\left|f^{\prime}(z)\right|+2 \varepsilon 1_{Z_{t}^{x} \geq 2 \varepsilon} \sup _{z \in[0,2 \varepsilon)}\left|f^{\prime}(z)\right| \sum_{k=0}^{\infty} 1_{\tau_{2 \varepsilon}^{k} \leq t}+1_{Z_{t}^{x}<2 \varepsilon}\left|f\left(Z_{t}^{x}\right)-f(x)\right| .
\end{aligned}
$$

We now take expectations ; since $Z^{x}$ leaves 0 continuously, we have that necessarily $Z_{\tau_{2 \varepsilon-}^{k}}>0$; thus, we may scale the law of the jumps at the jumping times $\tau_{2 \varepsilon}^{k}-$. Using this fact and scaling, gives

$$
\begin{aligned}
& \mathbb{E} \mid\left(f\left(Z_{t}^{x}\right)-f(x)\right)-\left(M_{t}^{f, \varepsilon}-M_{0}^{f, \varepsilon}\right) \\
& \quad-\beta_{1} \int_{0}^{t} f^{\prime}\left(Z_{s}^{x}\right) d s+\int_{0}^{t} d s \int_{0}^{\infty}[\Delta f]\left(Z_{s-}^{x}, a\right) 1_{Z_{s-}^{x}>0} \tilde{\nu}\left(Z_{s-}^{x}, d a\right) \mid \\
& \leq \mathbb{E}\left(1_{Z_{t}^{x} \geq 2 \varepsilon} \sum_{k=0}^{\infty}\left|f\left(Z_{\tau_{2 \varepsilon}^{k}-}^{x} J_{k}\right)-f\left(Z_{\tau_{2 \varepsilon}^{k}-}^{x}\right)\right| 1_{\left[Z_{\tau_{2 \varepsilon}^{k}-}^{x} J_{k}\right] \geq 2 \varepsilon} 1_{\tau_{2 \varepsilon}^{k} \leq t}\right) \\
& \quad+\sum_{k=0}^{\infty} \mathbb{E}\left(1_{\tau_{2 \varepsilon}^{k} \leq t} \int_{\left(\tau_{\varepsilon}^{k}, \tau_{2 \varepsilon}^{k}-\right]} d s \sup _{z \in[0,2 \varepsilon)}\left|f^{\prime}(z)\right| \int_{0}^{\infty} a 1_{Z_{s-}^{x}>0} \tilde{\nu}\left(Z_{s-}^{x}, d a\right) 1_{\tau_{2 \varepsilon}^{k} \leq t}\right) \\
& \quad+\left|\beta_{1}\right| t \sup _{z \in[0,2 \varepsilon)}\left|f^{\prime}(z)\right|+2 \varepsilon \mathbb{E}\left(1_{Z_{t}^{x} \geq 2 \varepsilon} \sup _{z \in[0,2 \varepsilon)}\left|f^{\prime}(z)\right| \sum_{k=0}^{\infty} 1_{\tau_{2 \varepsilon}^{k} \leq t}\right)+\mathbb{E}\left(1_{Z_{t}^{x}<2 \varepsilon}\left|f\left(Z_{t}^{x}\right)-f(x)\right|\right)
\end{aligned}
$$

where $\left(J_{k}\right)$ is a sequence of independent r.v. with density $\frac{1+\beta_{2}}{2 \beta_{2}}(1+a)^{-\gamma}$. 
Thus,

$$
\begin{aligned}
& \mathbb{E} \mid\left(f\left(Z_{t}^{x}\right)-f(x)\right)-\left(M_{t}^{f, \varepsilon}-M_{0}^{f, \varepsilon}\right) \\
& \quad-\beta_{1} \int_{0}^{t} f^{\prime}\left(Z_{s}^{x}\right) d s+\int_{0}^{t} d s \int_{0}^{\infty}[\triangle f]\left(Z_{s-}^{x}, a\right) 1_{Z_{s-}^{x}>0} \tilde{\nu}\left(Z_{s-}^{x}, d a\right) \mid \\
& \left.\leq(2 \varepsilon) \sup _{z \in[0,2 \varepsilon)}\left|f^{\prime}(z)\right| \mathbb{E}\left(1_{Z_{t}^{x} \geq 2 \varepsilon} \sum_{k=0}^{\infty}\left|J_{k}-1\right| 1_{\left[Z_{\tau_{2 \varepsilon}^{k}}^{x}\right.} J_{k}\right] \geq 2 \varepsilon 1_{\tau_{2 \varepsilon}^{k} \leq t}\right) \\
& \quad+\sum_{k=0}^{\infty} \mathbb{E}\left(1_{\tau_{2 \varepsilon}^{k} \leq t} \int_{\left(\tau_{\varepsilon}^{k}, \tau_{2 \varepsilon}^{k}-\right]} d s \sup _{z \in[0,2 \varepsilon)}\left|f^{\prime}(z)\right| \int_{0}^{\infty} d a \kappa \frac{a}{\left(Z_{s-}^{x}\right)^{2}}\left(1+\frac{a}{Z_{s-}^{x}}\right)^{-\gamma} 1_{Z_{s-}^{x}>0} 1_{\tau_{2 \varepsilon}^{k} \leq t}\right) \\
& \quad+\left|\beta_{1}\right| t \sup _{z \in[0,2 \varepsilon)}\left|f^{\prime}(z)\right|+2 \varepsilon \mathbb{E}\left(1_{Z_{t}^{x} \geq 2 \varepsilon} \sup _{z \in[0,2 \varepsilon)}\left|f^{\prime}(z)\right| \sum_{k=0}^{\infty} 1_{\tau_{2 \varepsilon}^{k} \leq t}\right)+\mathbb{E}\left(1_{Z_{t}^{x}<2 \varepsilon}\left|f\left(Z_{t}^{x}\right)-f(x)\right|\right) .
\end{aligned}
$$

A change of variable ensures that $\int_{0}^{\infty} \kappa \frac{a}{h^{2}}\left(1+\frac{a}{h}\right)^{-\gamma} 1_{h>0} d a<+\infty$ and does not depend on $h$. Moreover, from the fact that $\left(Z_{t}^{x}\right)$ is a process having only positive jumps and that its slope is $-\beta_{1}$, we deduce that for any $\varepsilon>0, \sum_{k=0}^{\infty} 1_{\tau_{2 \varepsilon}^{k} \leq t} \leq \frac{\beta_{1} t}{\varepsilon}$ a.s.

This implies that

$$
\begin{aligned}
& \mathbb{E} \mid\left(f\left(Z_{t}^{x}\right)-f(x)\right)-\left(M_{t}^{f, \varepsilon}-M_{0}^{f, \varepsilon}\right) \\
& \quad-\beta_{1} \int_{0}^{t} f^{\prime}\left(Z_{s}^{x}\right) d s+\int_{0}^{t} d s \int_{0}^{\infty}[\triangle f]\left(Z_{s-}^{x}, a\right) 1_{Z_{s-}^{x}>0} \tilde{\nu}\left(Z_{s-}^{x}, d a\right) \mid \\
& \leq C t \sup _{z \in[0,2 \varepsilon)}\left|f^{\prime}(z)\right|+C \sup _{z \in[0,2 \varepsilon)}\left|f^{\prime}(z)\right| \sum_{k=1}^{\infty} \mathbb{E}\left(1_{\tau_{2 \varepsilon}^{k} \leq t}\left(\tau_{2 \varepsilon}^{k}-\tau_{\varepsilon}^{k}\right)\right)+C \mathbb{P}\left(Z_{t}^{x}<2 \varepsilon\right) \\
& \leq C t \sup _{z \in[0,2 \varepsilon)}\left|f^{\prime}(z)\right|+C \sup _{z \in[0,2 \varepsilon)}\left|f^{\prime}(z)\right| \sum_{k=0}^{\infty} \mathbb{E}\left(1_{\tau_{2 \varepsilon}^{k} \leq t}\left(\tau_{2 \varepsilon}^{k+1}-\tau_{2 \varepsilon}^{k}\right)\right)+C \mathbb{P}\left(Z_{t}^{x}<2 \varepsilon\right) \\
& \leq C t \sup _{z \in[0,2 \varepsilon)}\left|f^{\prime}(z)\right|+C \mathbb{P}\left(Z_{t}^{x}<2 \varepsilon\right)
\end{aligned}
$$

where $C$ is some constant depending on $f$ and $x$ and possibly changing from line to line.

The last term is easily seen to tend to zero as $\varepsilon \searrow 0$ because of our assumptions on the function $f$. Since $\left(M_{t}^{f, \varepsilon}-M_{0}^{f, \varepsilon}\right)$ is of zero expectation, we deduce the Itô-Dynkin formula (47).

\subsection{Computation of the law of $Z_{1}^{0}$}

This section is devoted to the proof of the following result:

Theorem 5. Assume h.

For all $t>0$, the law of $Z_{t}^{0}$ has density

$$
p_{Z}(t, 0, y)=c_{1} \frac{1}{y}\left(\frac{t \beta_{1}}{y}\right)^{\gamma-1}\left(1+\frac{t \beta_{1}}{y}\right)^{1-\gamma-\xi^{\star}} \mathbb{I}_{y>0},
$$

where $c_{1}$ is defined by $c_{1}^{-1}:=\int_{0}^{\infty} z^{\gamma-2}(1+z)^{1-\gamma-\xi^{*}} \mathrm{~d} z=\Gamma(\gamma-1) \Gamma\left(\xi^{\star}\right) / \Gamma\left(\gamma+\xi^{\star}-1\right)$. 
The main ingredient in the proof is that because of the self-similarity of $Z$, the generator $A$ acts as a multiplier for Mellin'stransform. Let us recall that for $f:[0, \infty) \rightarrow \mathbb{R}$ one defines Mellin's transform of $f$ as

$$
\mathcal{M}[f](\xi)=\int_{0}^{\infty} x^{\xi-1} f(x) \mathrm{d} x
$$

for all $\xi \in \mathbb{C}$ such that the latter integral is well defined. It is clear that if $f$ is bounded and with exponential decay near $\infty$ then $\xi \mapsto \mathcal{M}[f](\xi)$ is well defined and holomorphic on the half plane $\{\xi \in \mathbb{C} \mid \operatorname{Re}(\xi)>0\}$.

For such functions $f$, we recall the four following properties which are easily derived from the definition of Mellin's transform:

$$
\begin{aligned}
\mathcal{M}[x \mapsto f(x(1+y))](\xi) & =(1+y)^{-\xi} \mathcal{M}[f](\xi), \text { for } \operatorname{Re}(\xi)>0 \\
\mathcal{M}[x \mapsto f(x) / x](\xi) & =\mathcal{M}[f](\xi-1), \text { for } \operatorname{Re}(\xi)>1 \\
\mathcal{M}\left[f^{\prime}\right](\xi) & =(1-\xi) \mathcal{M}[f](\xi-1), \text { if } f \in \mathcal{C}^{1}(0, \infty) \text { and } \operatorname{Re}(\xi)>1 \\
\mathcal{M}\left[x \mapsto x f^{\prime}(x)\right](\xi) & =-\xi \mathcal{M}[f](\xi), \text { if } f \in \mathcal{C}^{1}(0, \infty) \text { and } \operatorname{Re}(\xi)>0
\end{aligned}
$$

Proof. Choose $\xi$ with $\mathcal{R} e(\xi) \in\left(2, \frac{3}{2}+\frac{1}{2 \beta_{2}}\right)$. Applying the Itô-Dynkin formula $(47)$ to $f(y):=y^{\xi-1}$, we deduce,

$$
\begin{aligned}
\mathbb{E}\left(Z_{t}^{x}\right)^{\xi-1}= & x^{\xi-1}-\beta_{1}(\xi-1) \mathbb{E} \int_{0}^{t}\left(Z_{s}^{x}\right)^{\xi-2} \mathbb{I}_{Z_{s}^{x}>0} d s \\
& +\mathbb{E} \int_{0}^{t} d s \int_{0}^{\infty}\left(\left(Z_{s-}^{x}+a\right)^{\xi-1}-\left(Z_{s-}^{x}\right)^{\xi-1}\right) \tilde{\nu}\left(Z_{s-}^{x}, d a\right),
\end{aligned}
$$

where the moments above are finite (see Remark 2).

Performing the change of variable $\tilde{a}=\frac{a}{Z_{s}^{x}}$ gives

$$
\begin{aligned}
\mathbb{E}\left(Z_{t}^{x}\right)^{\xi-1}= & x^{\xi-1}-\beta_{1}(\xi-1) \mathbb{E} \int_{0}^{t}\left(Z_{s}^{x}\right)^{\xi-2} \mathbb{I}_{Z_{s}^{x}>0} d s \\
& +\mathbb{E} \int_{0}^{t} d s\left(Z_{s}^{x}\right)^{\xi-1} \mathbb{I}_{Z_{s}^{x}>0} \int_{0}^{\infty}\left((1+\tilde{a})^{\xi-1}-1\right) \frac{\kappa}{Z_{s}^{x}}(1+\tilde{a})^{-\gamma} d \tilde{a} \\
= & x^{\xi-1}-\beta_{1}(\xi-1) \mathbb{E} \int_{0}^{t}\left(Z_{s}^{x}\right)^{\xi-2} \mathbb{I}_{Z_{s}^{x}>0} d s \\
& +\mathbb{E} \int_{0}^{t} d s\left(Z_{s}^{x}\right)^{\xi-2} \mathbb{I}_{Z_{s}^{x}>0} \int_{0}^{\infty}\left((1+\tilde{a})^{\xi-1}-1\right) \kappa(1+\tilde{a})^{-\gamma} d \tilde{a}
\end{aligned}
$$

Since $\xi>2,\left(Z_{t}^{x}\right)^{\xi-2}=\left(Z_{t}^{x}\right)^{\xi-2} \mathbb{I}_{Z_{t}^{x}>\mathrm{o}}$ and

$$
\mathbb{E}\left(Z_{t}^{x}\right)^{\xi-1}=x^{\xi-1}-\beta_{1}(\xi-1) \int_{0}^{t} \mathbb{E}\left(Z_{s}^{x}\right)^{\xi-2} d s+\left(\frac{\kappa}{\gamma-\xi}-\frac{\kappa}{\gamma-1}\right) \int_{0}^{t} \mathbb{E}\left(Z_{s}^{x}\right)^{\xi-2} d s .
$$

For any $\lambda>0$, we set $w_{\lambda, x}(\xi):=\int_{0}^{\infty} \mathrm{e}^{-\lambda t} \mathbb{E}\left(Z_{t}^{x}\right)^{\xi-1} d t$. From an integration by parts we have,

$$
\begin{aligned}
& w_{\lambda, x}(\xi)=\frac{x^{\xi-1}}{\lambda}+\frac{w_{\lambda, x}(\xi-1)}{\lambda}\left(\frac{\kappa}{\gamma-\xi}-\frac{\kappa}{\gamma-1}-\beta_{1}(\xi-1)\right) \\
& \lambda w_{\lambda, x}(\xi)=x^{\xi-1}+w_{\lambda, x}(\xi-1)\left(\frac{\kappa}{\gamma-\xi}-\frac{\kappa}{\gamma-1}-\beta_{1}(\xi-1)\right) .
\end{aligned}
$$


Set $u_{\lambda, x}(\mathrm{~d} y):=\int_{0}^{\infty} \mathrm{e}^{-\lambda t} p_{Z}(t, x, \mathrm{~d} y) d t$.

We have for $x=0$,

$$
\begin{aligned}
\mathcal{M}\left[u_{\lambda, 0}\right](\xi) & =\int_{0}^{\infty} \mathrm{e}^{-\lambda t} \mathbb{E}\left(Z_{t}^{0}\right)^{\xi-1} \mathrm{~d} t \\
& =\int_{0}^{\infty} \mathrm{e}^{-\lambda t} \mathbb{E}\left(Z_{1}^{0}\right)^{\xi-1} t^{\xi-1} \mathrm{~d} t \\
& =\Gamma(\xi) \mathbb{E}\left(Z_{1}^{0}\right)^{\xi-1} .
\end{aligned}
$$

Fubini's theorem implies that

$$
\lambda \mathcal{M}\left[u_{\lambda, x}\right](\xi)=(\xi-1) \mathcal{M}\left[y \mapsto \frac{1}{y} \mathbb{I}_{0<y \leq x}\right](\xi)+\mathcal{M}\left[u_{\lambda, x}\right](\xi-1)\left(\frac{\kappa}{\gamma-\xi}-\frac{\kappa}{\gamma-1}-\beta_{1}(\xi-1)\right) .
$$

Let now $x \rightarrow 0$ and set $u_{\lambda}:=u_{\lambda, 0}$. We have,

$$
\lambda \mathcal{M}\left[u_{\lambda}\right](\xi)=\mathcal{M}\left[u_{\lambda}\right](\xi-1)\left(\frac{\kappa}{\gamma-\xi}-\frac{\kappa}{\gamma-1}-\beta_{1}(\xi-1)\right) .
$$

So that

$$
\lambda(\gamma-\xi)(\gamma-1) \mathcal{M}\left[u_{\lambda}\right](\xi)=-(\xi-1) \beta_{1}(\gamma-\xi)(\gamma-1) \mathcal{M}\left[u_{\lambda}\right](\xi-1)+\kappa(\xi-1) \mathcal{M}\left[u_{\lambda}\right](\xi-1),
$$

and

$$
\begin{aligned}
& \lambda \gamma(\gamma-1) \mathcal{M}\left[u_{\lambda}\right](\xi)-\lambda(\gamma-1) \xi \mathcal{M}\left[u_{\lambda}\right](\xi) \\
= & \beta_{1} \gamma(\gamma-1)(1-\xi) \mathcal{M}\left[u_{\lambda}\right](\xi-1)+\beta_{1}(\gamma-1) \xi(\xi-1) \mathcal{M}\left[u_{\lambda}\right](\xi-1)+\kappa(\xi-1) \mathcal{M}\left[u_{\lambda}\right](\xi-1) .
\end{aligned}
$$

Using the properties of Mellin's transform gives

$$
\begin{aligned}
& \lambda \gamma(\gamma-1) \mathcal{M}\left[u_{\lambda}\right](\xi)+\lambda(\gamma-1) \mathcal{M}\left[y \mapsto y u_{\lambda}^{\prime}(y)\right](\xi) \\
= & \beta_{1} \gamma(\gamma-1) \mathcal{M}\left[u_{\lambda}^{\prime}\right](\xi)+\beta_{1}(\gamma-1) \mathcal{M}\left[y \mapsto y u_{\lambda}^{\prime \prime}(y)\right](\xi)-\kappa \mathcal{M}\left[u_{\lambda}^{\prime}\right](\xi) .
\end{aligned}
$$

Dividing by $\gamma-1$ and using the fact that $\kappa /(\gamma-1)=\left(1-\beta_{1}\right) / 2$,

$$
\begin{aligned}
& \lambda \gamma \mathcal{M}\left[u_{\lambda}\right](\xi)+\lambda \mathcal{M}\left[y \mapsto y u_{\lambda}^{\prime}(y)\right](\xi) \\
= & \left(\beta_{1} \gamma-\left(1-\beta_{1}\right) / 2\right) \mathcal{M}\left[u_{\lambda}^{\prime}\right](\xi)+\beta_{1} \mathcal{M}\left[y \mapsto y u_{\lambda}^{\prime \prime}(y)\right](\xi) \\
= & \beta_{1}\left(2-\xi^{\star}\right) \mathcal{M}\left[u_{\lambda}^{\prime}\right](\xi)+\beta_{1} \mathcal{M}\left[y \mapsto y u_{\lambda}^{\prime \prime}(y)\right](\xi)
\end{aligned}
$$

with $\xi^{\star}:=\frac{1}{2 \beta_{1}}-\frac{1}{2 \beta_{2}}$. Inverting Mellin's transform gives (for $y>0$ ) :

$$
\beta_{1} y u_{\lambda}^{\prime \prime}(y)-\left(\lambda y+\beta_{1}\left(\xi^{\star}-2\right)\right) u_{\lambda}^{\prime}(y)-\lambda \gamma u_{\lambda}(y)=0 .
$$

Let us set

$$
v_{\lambda}(y):=\frac{\lambda}{\beta_{1}} u_{\lambda}\left(\frac{\beta_{1} y}{\lambda}\right) .
$$


Then, $v_{\lambda}$ is solution of the Kummer's equation (for $y>0$ ) :

$$
y v_{\lambda}^{\prime \prime}(y)+\left(\left(2-\xi^{\star}\right)-y\right) v_{\lambda}^{\prime}(y)-\gamma v_{\lambda}(y)=0
$$

From the theory of solutions of Kummer's equation, if $M$ and $U$ are the confluent geometric functions, we may deduce that there exist two constants $b_{1}(\lambda)$ and $b_{2}(\lambda)$ such that for $y>0$ :

$$
v_{\lambda}(y)=b_{1}(\lambda) U\left(\gamma, 2-\xi^{\star}, y\right)+b_{2}(\lambda) M\left(\gamma, 2-\xi^{\star}, y\right)
$$

Here $M$ and $U$ denote the confluent hypergeometric functions (the functions $M$ and $U$ are defined by formulas 13.1.2 and 13.1.3 in chapter 13 of [1], and see also formula 13.2.6 of [1] for an integral representation of $U$ ). So that

$$
u_{\lambda}(y)=b_{1}(\lambda) \frac{\beta_{1}}{\lambda} \int_{0}^{\infty} \mathrm{e}^{-\frac{\lambda y t}{\beta_{1}}} t^{\gamma-1}(1+t)^{1-\gamma-\xi^{\star}} \mathrm{d} t+b_{2}(\lambda) M\left(\gamma, 2-\xi^{\star}, y\right) .
$$

$M\left(\gamma, 2-\xi^{\star}, y\right)$ tends to $\infty$ with $y$ (see [1] 13.1.4. p.504), so that necessarily $b_{2}(\lambda)=0$. Moreover, the homogeneity of $u_{\lambda}$ ensures that $b_{1}(\lambda) \propto \lambda$. Identification of the Laplace's transform ends the proof.

Remark 4. One may want to see if the above formula satisfies (at least formally) the Fokker-Planck forward equation, which in our case may be written as

$$
\left\{\begin{array}{l}
\frac{\partial}{\partial t} p_{Z}(t, 0, y)=\mathfrak{A}^{*} p_{Z}(t, 0, y) \quad(y>0), \\
p_{Z}(t, 0, y) \mathrm{d} y \underset{t \searrow 0}{\longrightarrow} \delta_{0}(\mathrm{~d} y) .
\end{array}\right.
$$

Here $\mathfrak{A}^{*}$ denotes the formal adjoint of $\mathfrak{A}$. It is defined for any $\varphi \in \mathcal{C}^{1}((0, \infty))$ such that $\mathfrak{A}^{*} \varphi<+\infty$ by

$$
\begin{aligned}
\mathfrak{A}^{*} \varphi(y) & =\beta_{1} \varphi^{\prime}(y)-\int_{0}^{\infty}\left(\varphi(y) \frac{\kappa}{y^{2}}\left(1+\frac{a}{y}\right)^{-\gamma}-\varphi(y-a) \frac{\kappa}{(y-a)^{2}}\left(1+\frac{a}{y-a}\right)^{-\gamma} \mathbf{1}_{0<a<y}\right) \mathrm{d} a \\
& =\beta_{1} \varphi^{\prime}(y)-\kappa \int_{0}^{\infty}\left(\varphi(y) y^{\gamma-2}(y+a)^{-\gamma}-\varphi(y-a) y^{-\gamma}(y-a)^{\gamma-2} \mathbf{1}_{0<a<y}\right) \mathrm{d} a \\
& =\beta_{1} \varphi^{\prime}(y)-\kappa\left(\frac{\varphi(y)}{(\gamma-1) y}-\int_{0}^{\infty} \varphi(y-a) y^{-\gamma}(y-a)^{\gamma-2} \mathbf{1}_{0<a<y} \mathrm{~d} a\right) \\
& =\beta_{1} \varphi^{\prime}(y)-\kappa\left(\int_{0}^{\infty}[\varphi(y)-\varphi(y-a)] y^{-\gamma}(y-a)^{\gamma-2} \mathbf{1}_{0<a<y} \mathrm{~d} a\right)
\end{aligned}
$$

where the last lines come from the fact that $\gamma-1>1$ (because $\left.\beta_{2}<1\right)$ and $\int_{0}^{y}(y-a)^{\gamma-2} \mathrm{~d} a=\frac{y^{\gamma}}{(\gamma-1) y}$. Consequently, let us denote $\Gamma$ the function $(t, y) \mapsto \frac{\beta_{1}}{y}\left(\frac{t \beta_{1}}{y}\right)^{\gamma-1}\left(\frac{t \beta_{1}}{y}+1\right)^{1-\gamma-\xi^{\star}}=c_{1}^{-1} p_{Z}(t, 0, y)$.

$$
\begin{aligned}
\frac{\partial \Gamma}{\partial t}(t, y) & =\left(\frac{\beta_{1}}{y}\right)^{2}\left(\frac{t \beta_{1}}{y}\right)^{\gamma-2}\left(\frac{t \beta_{1}}{y}+1\right)^{-\left(\gamma+\xi^{\star}\right)}\left[(\gamma-1)\left(\frac{t \beta_{1}}{y}+1\right)+\left((1-\gamma)-\xi^{\star}\right) \frac{t \beta_{1}}{y}\right] \\
=\left(\frac{\beta_{1}}{y}\right)^{2}\left(\frac{t \beta_{1}}{y}\right)^{\gamma-2}\left(\frac{t \beta_{1}}{y}+1\right)^{-\left(\gamma+\xi^{\star}\right)}\left((\gamma-1)-\xi^{\star} \frac{t \beta_{1}}{y}\right) . & \\
\beta_{1} \frac{\partial \Gamma}{\partial y}(t, y) & =\left(\frac{\beta_{1}}{y}\right)^{2}\left(\frac{t \beta_{1}}{y}\right)^{\gamma-1}\left(\frac{t \beta_{1}}{y}+1\right)^{-\left(\gamma+\xi^{\star}\right)}\left[-\gamma\left(\frac{t \beta_{1}}{y}+1\right)-\left(1-\gamma-\xi^{\star}\right) \frac{t \beta_{1}}{y}\right] \\
& =\left(\frac{\beta_{1}}{y}\right)^{2}\left(\frac{t \beta_{1}}{y}\right)^{\gamma-1}\left(\frac{t \beta_{1}}{y}+1\right)^{-\left(\gamma+\xi^{\star}\right)}\left[\left(\xi^{\star}-1\right) \frac{t \beta_{1}}{y}-\gamma\right] .
\end{aligned}
$$




$$
\begin{aligned}
\frac{\kappa}{\gamma-1} \frac{\Gamma(t, y)}{y} & =\frac{\kappa}{\gamma-1} \frac{\beta_{1}}{y^{2}}\left(\frac{t \beta_{1}}{y}\right)^{\gamma-1}\left(\frac{t \beta_{1}}{y}+1\right)^{1-\gamma-\xi^{\star}} . \\
\kappa \int_{0}^{y} \Gamma(t, y-a) y^{-\gamma}(y-a)^{\gamma-2} \mathrm{~d} a & =\kappa \int_{0}^{y} \frac{\beta_{1}}{y-a}\left(\frac{t \beta_{1}}{y-a}\right)^{\gamma-1}\left(\frac{t \beta_{1}}{y-a}+1\right)^{1-\gamma-\xi^{\star}} y^{-\gamma}(y-a)^{\gamma-2} \mathrm{~d} a \\
& =\kappa \beta_{1}\left(t \beta_{1}\right)^{\gamma-1} y^{-\gamma} \int_{0}^{y}\left(\frac{t \beta_{1}}{y-a}+1\right)^{1-\gamma-\xi^{\star}}(y-a)^{-2} \mathrm{~d} a \\
& =\kappa \beta_{1}\left(t \beta_{1}\right)^{\gamma-1} y^{-\gamma} \int_{0}^{y}\left(\frac{t \beta_{1}}{u}+1\right)^{1-\gamma-\xi^{\star}}(u)^{-2} \mathrm{~d} u \\
& =\kappa t^{\gamma-2} \beta_{1}^{\gamma-1} y^{-\gamma} \int_{\frac{t \beta_{1}}{y}}^{+\infty}(x+1)^{1-\gamma-\xi^{\star}} \mathrm{d} x \\
& =-\frac{\kappa}{2-\gamma-\xi^{\star}} \frac{\beta_{1}}{y^{2}}\left(\frac{t \beta_{1}}{y}\right)^{\gamma-2}\left(\frac{t \beta_{1}}{y}+1\right)^{2-\gamma-\xi^{\star}} .
\end{aligned}
$$

Hence,

$$
\begin{aligned}
& {\left[\frac{\partial}{\partial t}-\mathfrak{A}^{*}\right] \Gamma(t, y)=\frac{1}{y^{2}}\left(\frac{t \beta_{1}}{y}\right)^{\gamma-2}\left(\frac{t \beta_{1}}{y}-1\right)^{-\gamma-\xi^{\star}} \times} \\
& \left(\beta_{1}^{2}\left((\gamma-1)-\xi^{\star} \frac{t \beta_{1}}{y}\right)-\beta_{1}^{2} \frac{t \beta_{1}}{y}\left(\left(\xi^{\star}-1\right) \frac{t \beta_{1}}{y}-\gamma\right)+\frac{\kappa \beta_{1}}{\gamma-1}\left(\frac{t \beta_{1}}{y}\left(\frac{t \beta_{1}}{y}+1\right)\right)+\frac{\kappa \beta_{1}}{2-\gamma-\xi^{\star}}\left(\frac{t \beta_{1}}{y}+1\right)^{2}\right) .
\end{aligned}
$$

One may easily check at once that

$$
\begin{array}{r}
\beta_{1}^{2}\left(1-\xi^{\star}\right)+\frac{\kappa \beta_{1}}{\gamma-1}+\frac{\kappa \beta_{1}}{2-\gamma-\xi^{\star}}=0, \\
\beta_{1}^{2}(\gamma-1)+\frac{\kappa \beta_{1}}{2-\gamma-\xi^{\star}}=0, \\
-\beta_{1}^{2} \xi^{\star}+\beta_{1}^{2} \gamma+\frac{\kappa \beta_{1}}{\gamma-1}+\frac{2 \kappa \beta_{1}}{2-\gamma-\xi^{\star}}=0 .
\end{array}
$$

So that

$$
\left[\frac{\partial}{\partial t}-\mathfrak{A}^{*}\right] \Gamma(t, y)=0
$$

Remark 5. (A remark about the law of $Z$ starting from $x>0$ )

Set $u_{\lambda, x}(y):=\int_{0}^{\infty} \mathrm{e}^{-\lambda t} p_{Z}(t, x, y) d t$. We may retake the previous computations without letting $x$ tend to 0.

Then Fubini's theorem implies that

$$
\lambda \mathcal{M}\left[u_{\lambda, x}\right](\xi)=(\xi-1) \mathcal{M}\left[y \mapsto \frac{1}{y} \mathbb{I}_{0<y \leq x}\right](\xi)+\mathcal{M}\left[u_{\lambda, x}\right](\xi-1)\left(\frac{\kappa}{\gamma-\xi}-\frac{\kappa}{\gamma-1}-\beta_{1}(\xi-1)\right)
$$

Using then the properties of Mellin's transform, we arrive after tedious calculations at

$$
\begin{aligned}
& \hat{u}_{\lambda, x}(\zeta) \\
& =\left(\hat{u}_{\lambda, x}\left(\zeta_{0}\right)+\int_{\zeta_{0}}^{\zeta}\left((\gamma-1)\left(1-\mathrm{e}^{-2 i \pi x \theta}\right)-2 i \pi \theta x \mathrm{e}^{-2 i \pi x \theta}+\beta_{1}\left(1-\xi^{\star}\right) u_{\lambda, x}(0)\right) \theta^{-\gamma}\left(2 i \pi \beta_{1} \theta-\lambda\right)^{\xi^{\star}+\gamma-2} \mathrm{~d} \theta\right) \\
& \quad \times\left(\zeta^{1-\gamma}\left(2 i \pi \beta_{1} \zeta-\lambda\right)^{\xi^{\star}-(1-\gamma)}-\zeta_{0}^{1-\gamma}\left(2 i \pi \beta_{1} \zeta_{0}-\lambda\right)^{\xi^{\star}-(1-\gamma)}\right) .
\end{aligned}
$$

where $\hat{u}_{\lambda, x}$ stands for the Fourier transform of $u_{\lambda, x}$. Unfortunately this formula does not seem easily invertible. 


\subsection{Mean long time behavior}

From this expression of $p_{Z}(t, 0, y) \mathrm{d} y$ we deduce the following results.

Proposition 5. Assume h.

Set $\pi(y)=\frac{c_{1}}{\beta_{1}^{\xi^{\star}}} \frac{1}{y^{1-\xi^{\star}}}$ defined for $y>0$. Then, for any fixed $y>0$,

$$
\left|t^{\xi^{\star}} p_{Z}(t, 0, y)-\pi(y)\right| \underset{t \rightarrow+\infty}{\longrightarrow} 0
$$

Proposition 6. Assume h.

For any real Borel function $f$ defined on $[0, \infty)$ such that $\int_{0}^{\infty} \frac{f(y)}{y^{1-\xi^{\star}}}<\infty$,

$$
\frac{1}{t^{1-\xi^{\star}}} \int_{0}^{t} \mathbb{E} f\left(Z_{s}^{0}\right) \mathrm{d} s \underset{t \rightarrow+\infty}{\longrightarrow} \frac{1}{1-\xi^{\star}} \int_{0}^{+\infty} f(y) \pi(\mathrm{d} y)
$$

Proof. As already noticed, under hypothesis $\mathfrak{h}$, we have that $0<1-\xi^{*}<1$. It is not hard to see that $\int_{0}^{t} p_{Z}(s, 0, y) \mathrm{d} s=O\left(t^{1-\xi^{\star}}\right)$ and is strictly increasing as $t$ tends to $\infty$. Moreover, from the Fubini-Tonnelli theorem and a simple change of variable, we have that

$$
\begin{aligned}
\frac{1}{t^{1-\xi^{\star}}} \int_{0}^{t} \mathbb{E} f\left(Z_{s}^{0}\right) \mathrm{d} s & =\frac{1}{t^{1-\xi^{\star}}} \int_{0}^{t} \int_{0}^{+\infty} f(y) p_{Z}(s, 0, y) \mathrm{d} y \mathrm{~d} s \\
& =\int_{0}^{+\infty} f(y) \frac{c_{1} \beta_{1}^{-\xi^{\star}}}{y^{1-\xi^{\star}}}\left(\frac{y}{t \beta_{1}}\right)^{1-\xi^{\star}}\left(\int_{0}^{\frac{t \beta_{1}}{y}} u^{\gamma-1}(1+u)^{1-\gamma-\xi^{\star}} \mathrm{d} u\right) \mathrm{d} y \\
& \underset{t \rightarrow+\infty}{\longrightarrow} \int_{0}^{+\infty} f(y) \pi(\mathrm{d} y)
\end{aligned}
$$

with $\pi(\mathrm{d} y):=c_{1} c_{2} \beta_{1}^{-\xi^{\star}} \frac{\mathrm{d} y}{y^{1-\xi^{\star}}}, c_{2}:=\frac{1}{1-\xi^{\star}}\left(=\lim _{X \rightarrow+\infty} \frac{1}{X^{1-\xi^{\star}}} \int_{0}^{X} u^{\gamma-1}(1+u)^{1-\gamma-\xi^{\star}} \mathrm{d} u\right)$ and where the last line comes from Lebesgue's domination theorem.

We may check that $\pi(\mathrm{d} y)$ thus defined is in fact an invariant measure for $\left(Z_{t}\right)_{t \geq 0}$ meaning that

$$
\mathfrak{A}^{*} \pi(\mathrm{d} y)=0
$$

\section{Excursions of the difference process}

From the result of Theorem 2, we have that

$$
\lim _{x \rightarrow 0+} \frac{\mathbb{E}^{x}\left(1-\exp \left(-U^{\star}\right)\right)}{x^{1-\xi^{\star}}}=\frac{1}{\mathbf{b}\left(1-\xi^{\star}, \frac{1-\beta_{1}}{2 \beta_{1}}\right)} \int_{0}^{\infty}\left(1-\mathrm{e}^{-u}\right) \beta_{1}\left(\beta_{1} u\right)^{\xi^{\star}-2} \mathrm{~d} u \in(0, \infty)
$$

where $\mathbf{b}(a, b)=\int_{0}^{1} u^{a-1}(1-u)^{b-1} \mathrm{~d} u=\frac{\Gamma(a) \Gamma(b)}{\Gamma(a+b)}$.

Since $\left(Z^{x}\right)$ has been shown to be the positive self-similar recurrent extension of $\left(Z^{x, \dagger}\right)$ that leaves 0 continuously, the theory of self-similar recurrent extensions asserts from (54) that the excursion measure $\mathrm{n}$ is self-similar 
with index $1-\xi^{\star}$. In turn, the self-similarity of index $1-\xi^{\star}$ for the excursion measure $\mathrm{n}$ of the process $\left(Z_{t}^{x}\right)_{t \geq 0}$ implies that there exists a constant $c$ (namely, $c=\mathrm{n}(R(\mathrm{e})>1)$ ) such that

$$
\mathrm{n}(R(\mathrm{e})>t)=c t^{-\left(1-\xi^{\star}\right)}, \quad t>0 .
$$

(see iii) Lemma 2 in [20]). In particular, $\mathrm{n}\left(1-\mathrm{e}^{-\lambda R(\mathrm{e})}\right)=\lambda \int_{0}^{\infty} \mathrm{e}^{-\lambda \theta} \mathrm{n}(R(\mathrm{e})>\theta) \mathrm{d} \theta \propto \lambda \int_{0}^{\infty} \mathrm{e}^{-\lambda \theta} \theta^{\xi^{\star}-1} \mathrm{~d} \theta$ (from the previous) and we get that

$$
\mathrm{n}\left(1-\mathrm{e}^{-\lambda R(\mathrm{e})}\right) \propto \lambda^{1-\xi^{\star}}, \quad \lambda>0 .
$$

In particular, this implies that the Levy measure $\nu(\mathrm{d} t)$ of the subordinator $\left(\varsigma_{t}^{0}, \mathbb{P}^{0}\right)$ takes the form

$$
\nu(\mathrm{d} t)=c\left(1-\xi^{\star}\right) \frac{\mathrm{d} t}{t^{2-\xi^{\star}}}
$$

\subsection{Entrance law}

Let us denote

$$
Q_{u} f(x)=\mathbb{E}_{x}\left(f\left(Z_{u}^{\dagger, x}\right) \mathbb{I}_{u<U^{\star}(x)}\right) .
$$

We have

$$
Q_{u}(x, A)=Q_{a u}(a x, a A) .
$$

Let $\left(\mathrm{n}_{t}(\mathrm{~d} y)\right)_{t>0}:=\left(\mathrm{n}\left(\mathbb{I}_{e_{t} \in \mathrm{d} y} \mathbb{I}_{t \leq R(\mathrm{e})}\right)\right)_{t>0}$ denote the family of entrance laws satisfying $\mathrm{n}_{t} Q_{s}=\mathrm{n}_{t+s}(t, s>0)$ and related to $\mathrm{n}$. The family of entrance measures $\left(\mathrm{n}_{t}(\mathrm{~d} y)\right)_{t>0}$ may then be described in terms of the underlying Levy process thanks to the result of [21] (See Theorem 2. formula (3)), which in our case and with our notations reads

$$
\mathrm{n}_{t}(f)=\frac{1}{t^{1-\xi^{\star}} \Gamma\left(1-\xi^{\star}\right) \mathbb{E}^{\natural}\left(J^{-\xi^{\star}}\right)} \mathbb{E}^{\natural}\left(f\left(\frac{t}{J}\right) J^{-\xi^{\star}}\right) \text {, with } J:=\int_{0}^{\infty} \mathrm{e}^{-H_{s}^{0}} \mathrm{~d} s
$$

and where $\mathbb{E}^{\natural}$ denotes the Lamperti transform of the canonical Lévy process under the $h$-transform probability measure (with respect to $h: y \mapsto \mathrm{e}^{\left(1-\xi^{\star}\right) y}$ ) of the law of $\left(H_{t}\right)_{t \geq 0}$.

We shall prove the following result,

Theorem 6. Assume h.

The family of entrance laws $\left(\mathrm{n}_{t}(\mathrm{~d} y)\right)_{t>0}$ related to the description of $\mathrm{n}(\mathrm{de})$ are given by

$$
\mathrm{n}_{t}(\mathrm{~d} y) \propto t^{\gamma+\xi^{\star}-2}\left(y+\beta_{1} t\right)^{-\gamma} \mathrm{d} y .
$$

Proof. From the theory of self-similar recurrent extensions (Master formula applied firstly for $V_{s}=\mathrm{e}^{-\lambda s}$ and $F=\int_{0}^{R(\mathrm{e})} \mathrm{e}^{-\lambda u} f\left(\mathrm{e}_{u}\right) \mathrm{d} u$ and secondly to for $\left.f \equiv 1\right)$, for any positive Borel function, we have

$$
u[f](\lambda):=\int_{0}^{\infty} \mathrm{e}^{-\lambda t}\left(\int_{0}^{\infty} f(y) p_{Z}(t, 0, y) \mathrm{d} y\right) \mathrm{d} t=\frac{\int_{0}^{\infty} \mathrm{e}^{-\lambda t} \mathrm{n}\left(f\left(\mathrm{e}_{t}\right) \mathbb{I}_{t \leq R(\mathrm{e})}\right) \mathrm{d} t}{\mathrm{n}\left(1-\mathrm{e}^{-\lambda R(\mathrm{e})}\right)} .
$$

A notable consequence is that using (55)

$$
\lambda^{1-\xi^{\star}} u[f](\lambda)=\frac{1}{\lambda^{\left(\xi^{\star}-1\right)+1}}\left(\lambda u_{\lambda, 0}(f)\right) \propto \int_{0}^{\infty} \mathrm{e}^{-\lambda t} \mathrm{n}\left(f\left(\mathrm{e}_{t}\right) \mathbb{I}_{t \leq R(\mathrm{e})}\right) \mathrm{d} t:=\mathcal{L}\left[\mathrm{n}_{t}(f)\right](\lambda) .
$$


This decomposition allows to invert the Laplace transforms in order do guarantee the integrability of the integrands ; and for any $t>0$,

$$
\int_{0}^{t}(t-s)^{\xi^{\star}-1} \frac{\partial}{\partial s}\left(\int_{0}^{\infty} f(y) p_{Z}(s, 0, y) \mathrm{d} y\right) \mathrm{d} s \propto \mathrm{n}\left(f\left(\mathrm{e}_{t}\right) \mathbb{I}_{t \leq R(\mathrm{e})}\right) .
$$

From the previous computation, for any positive function $f \in \mathcal{C}^{1}([0, \infty))$ such that $f \in L^{1}(\pi)$, we get from Fokker-Planck's equation that

$$
\begin{aligned}
\mathrm{n}_{t}(f) & \propto \int_{0}^{\infty}\left(\int_{0}^{t}(t-s)^{\xi^{\star}-1} \mathfrak{A}^{*} p_{Z}(s, 0, y) \mathrm{d} s\right) f(y) \mathrm{d} y \\
& \propto \int_{0}^{\infty} \mathfrak{A}^{*}\left(\int_{0}^{t}(t-s)^{\xi^{\star}-1} p_{Z}(s, 0, y) \mathrm{d} s\right) f(y) \mathrm{d} y \\
& \propto \int_{0}^{\infty}\left(\int_{0}^{t}(t-s)^{\xi^{\star}-1} p_{Z}(s, 0, y) \mathrm{d} s\right) \mathfrak{A} f(y) \mathrm{d} y \\
& \propto \int_{0}^{\infty}\left(\int_{0}^{t}(t-s)^{\xi^{\star}-1} s^{\gamma-1}\left(y+s \beta_{1}\right)^{1-\gamma-\xi^{\star}} \mathrm{d} s\right) \mathfrak{A} f(y) \pi(\mathrm{d} y) .
\end{aligned}
$$

So that

$$
\begin{aligned}
\mathcal{L}\left[\mathrm{n}_{s}(f)\right](\lambda) & \propto \frac{1}{\lambda^{\xi^{\star}}} \mathcal{L}\left[\left\langle s^{\gamma-1}\left(.+s \beta_{1}\right)^{1-\gamma-\xi^{\star}}, \mathfrak{A} f\right\rangle_{\pi}\right](\lambda) \\
& \propto \frac{1}{\lambda^{\xi^{\star}}}\left\langle\mathcal{L}\left[s^{\gamma-1}\left(.+s \beta_{1}\right)^{1-\gamma-\xi^{\star}}\right](\lambda), \mathfrak{A} f\right\rangle_{\pi} .
\end{aligned}
$$

Note that using the change of variable $\theta=s \beta_{1} / y$ in the integral gives,

$$
\begin{aligned}
\frac{1}{\lambda^{\star}} \int_{0}^{\infty} \mathrm{e}^{-\lambda s} s^{\gamma-1}\left(y+s \beta_{1}\right)^{1-\gamma-\xi^{\star}} \mathrm{d} s & =\frac{y^{1-\xi^{\star}}}{\beta_{1}^{\gamma} \lambda^{\star}} \int_{0}^{\infty} \mathrm{e}^{-\lambda \frac{y \theta}{\beta_{1}}} \theta^{\gamma-1}(1+\theta)^{1-\gamma-\xi^{\star}} \mathrm{d} \theta \\
& =\frac{y^{1-\xi^{\star}}}{\beta_{1}^{\gamma}} \int_{0}^{\infty} \mathrm{e}^{-\lambda \frac{y \theta}{\beta_{1}}}(\lambda \theta)^{\gamma-1}(\lambda+\lambda \theta)^{1-\gamma-\xi^{\star}} \mathrm{d} \theta \\
& \propto \frac{y^{1-\xi^{\star}}}{\lambda} \int_{0}^{\infty} \mathrm{e}^{-u y} u^{\gamma-1}\left(\lambda+u \beta_{1}\right)^{1-\gamma-\xi^{\star}} \mathrm{d} u
\end{aligned}
$$

Hence,

$$
\begin{aligned}
\mathcal{L}\left[\mathrm{n}_{s}(f)\right](\lambda) & \propto \frac{1}{\lambda^{\star}}\left\langle\mathcal{L}\left[s^{\gamma-1}\left(.+s \beta_{1}\right)^{1-\gamma-\xi^{\star}}\right](\lambda), \mathfrak{A} f\right\rangle_{\pi} \\
& \propto \frac{1}{\lambda}\left\langle y \mapsto \mathcal{L}\left[s^{\gamma-1}\left(\lambda+s \beta_{1}\right)^{1-\gamma-\xi^{\star}}\right](y), \mathfrak{A} f\right\rangle \\
& \propto \frac{1}{\lambda} \int_{0}^{\infty} s^{\gamma-1}\left(\lambda+s \beta_{1}\right)^{1-\gamma-\xi^{\star}}\left\langle y \mapsto \mathfrak{A}^{*}\left(\mathrm{e}^{-y s}\right), f\right\rangle \mathrm{d} s .
\end{aligned}
$$

Note that the inverse Laplace transform of $\lambda \mapsto\left(\lambda+s \beta_{1}\right)^{1-\gamma-\xi^{\star}}$ is $t \mapsto \frac{1}{\Gamma\left(\gamma+\xi^{\star}-1\right)} t^{\gamma+\xi^{\star}-2} \mathrm{e}^{-t s \beta_{1}}$. Inverting Laplace's transforms, we finally find that

$$
\mathrm{n}_{t}(f) \propto \int_{0}^{\infty} s^{\gamma-1}\left\langle\mathfrak{A}^{*}\left(\mathrm{e}^{-y s}\right), f\right\rangle\left(\int_{0}^{t} \mathrm{e}^{-u s \beta_{1}} u^{\gamma+\xi^{\star}-2} \mathrm{~d} u\right) \mathrm{d} s,
$$

and

$$
\begin{aligned}
\mathrm{n}_{t}(\mathrm{~d} y) & \propto\left(\int_{0}^{t} u^{\gamma+\xi^{\star}-2}\left[\mathfrak{A}^{*}\left(\int_{0}^{\infty} s^{\gamma-1} \mathrm{e}^{-\left(y+u \beta_{1}\right) s} \mathrm{~d} s\right)\right] \mathrm{d} u\right) \mathrm{d} y \\
& \propto \int_{0}^{t} u^{\gamma+\xi^{\star}-2} \mathfrak{A}^{*}\left(\left(y+u \beta_{1}\right)^{-\gamma}\right) \mathrm{d} u \mathrm{~d} y .
\end{aligned}
$$


Let us compute $\mathfrak{A}^{*}\left(y \mapsto\left(y+u \beta_{1}\right)^{-\gamma}\right)$. We get that

$$
\begin{aligned}
\mathfrak{A}^{*}\left(\left(y+u \beta_{1}\right)^{-\gamma}\right) & =-\beta_{1} \gamma\left(y+u \beta_{1}\right)^{-\gamma-1}-\frac{\kappa}{\gamma-1} y^{-1}\left(y+u \beta_{1}\right)^{-\gamma}+\kappa y^{-\gamma} \int_{0}^{y}\left(\theta+u \beta_{1}\right)^{-\gamma} \theta^{\gamma-2} \mathrm{~d} \theta \\
& =-\beta_{1} \gamma\left(y+u \beta_{1}\right)^{-\gamma-1}-\frac{\kappa}{\gamma-1} y^{-1}\left(y+u \beta_{1}\right)^{-\gamma}+\kappa y^{-\gamma} \int_{0}^{y}\left(1+\frac{u \beta_{1}}{\theta}\right)^{-\gamma} \theta^{-2} \mathrm{~d} \theta \\
& =-\beta_{1} \gamma\left(y+u \beta_{1}\right)^{-\gamma-1}-\frac{\kappa}{\gamma-1} y^{-1}\left(y+u \beta_{1}\right)^{-\gamma}+\frac{\kappa}{\gamma-1} y^{-1}\left(u \beta_{1}\right)^{-1}\left(y+u \beta_{1}\right)^{1-\gamma} \\
& =\left(y+u \beta_{1}\right)^{-\gamma-1}\left(\frac{\kappa}{\gamma-1} \frac{y}{u \beta_{1}}+\left(\frac{\kappa}{\gamma-1}-\beta_{1} \gamma\right)\right)
\end{aligned}
$$

which in turn implies that

$$
\mathrm{n}_{t}(\mathrm{~d} y) \propto \int_{0}^{t} u^{\gamma+\xi^{\star}-3}\left(y+u \beta_{1}\right)^{-\gamma-1}\left(\frac{\kappa}{\gamma-1}\left(y+u \beta_{1}\right)-\beta_{1}^{2} \gamma u\right) \mathrm{d} u \mathrm{~d} y .
$$

Note that $\gamma+\xi^{\star}-3>-1 \Leftrightarrow-3 / 2+1 /\left(2 \beta_{1}\right)>-1 \Leftrightarrow 1 /\left(2 \beta_{1}\right)>1 / 2$ guaranteed by the assumption $0<\beta_{1}<1$, and so the above integral is definite.

Moreover, setting $\delta:=\frac{\kappa}{(\gamma-1) \beta_{1}}-1=\frac{1-3 \beta_{1}}{2 \beta_{1}}$. We see that

$$
\mathrm{n}_{t}(\mathrm{~d} y) \propto \beta_{1} \int_{0}^{t} u^{\gamma+\xi^{\star}-3-\delta} \frac{\partial}{\partial u}\left(u^{\delta+1}\left(y+\beta_{1} u\right)^{-\gamma}\right) \mathrm{d} u \mathrm{~d} y .
$$

But $\gamma+\xi^{\star}-3=-3 / 2+1 /\left(2 \beta_{1}\right)=\delta$. So $\gamma+\xi^{\star}-3-\delta=0$ and finally

$$
\mathrm{n}_{t}(\mathrm{~d} y) \propto t^{\frac{1-\beta_{1}}{2 \beta_{1}}}\left(y+\beta_{1} t\right)^{-\gamma} \mathrm{d} y .
$$

Remark 6. Let us check that

$$
\int_{0}^{\infty} \mathrm{n}_{t}(\mathrm{~d} y) \mathrm{d} t \propto \pi(\mathrm{d} y)
$$

as announced by the theory (see for example) : indeed, note that our assumption $\mathfrak{h}$ implies that $\frac{1-\beta_{1}}{2 \beta_{1}}>-1$ and $\gamma-\frac{1-\beta_{1}}{2 \beta_{1}}=2-\xi^{\star}>1$, so that the integral below is definite, and we have that

$$
\begin{aligned}
\int_{0}^{\infty} \mathrm{n}_{t}(\mathrm{~d} y) \mathrm{d} t & \propto \int_{0}^{\infty} t^{\frac{1-\beta_{1}}{2 \beta_{1}}}\left(y+\beta_{1} t\right)^{-\gamma} \mathrm{d} t \propto \int_{0}^{\infty} t^{\frac{1-\beta_{1}}{2 \beta_{1}}} t^{-\gamma}\left(\frac{y}{t}+\beta_{1}\right)^{-\gamma} \mathrm{d} t \\
& \propto \pi(\mathrm{d} y) \int_{0}^{\infty} \frac{1}{y}\left(\frac{y}{t}\right)^{2-\xi^{\star}}\left(\frac{y}{t}+\beta_{1}\right)^{-\gamma} \mathrm{d} t \propto \pi(\mathrm{d} y) \int_{0}^{\infty} \frac{1}{y}\left(\frac{y}{t}\right)^{2-\xi^{\star}}\left(\frac{y}{t}+\beta_{1}\right)^{-\gamma} \mathrm{d} t \\
& \propto\left(\int_{0}^{\infty} \theta^{-\xi^{\star}}\left(\theta+\beta_{1}\right)^{-\gamma} \mathrm{d} t\right) \pi(\mathrm{d} y) .
\end{aligned}
$$

\subsection{Last exit decomposition before time $t=1$ and Azema's projection}

Let us introduce

$$
g_{t}:=\sup \left(s \leq t: Z_{s}^{0}=0\right)
$$

and the subfiltration $\left(\check{\mathcal{F}}_{t}\right):=\left(\sigma\left\{H_{g_{t}}: H\right.\right.$ ranges through $\left(\mathcal{F}_{t}\right)$ optional processes $\left.\}\right)$. 
From (59), we have (see Theorem 7.2 in [23])

$$
\begin{aligned}
p_{Z}(1,0, y) & \propto \int_{0}^{1}(1-s)^{-\xi^{\star}} s^{\gamma+\xi^{\star}-2}\left(y+\beta_{1} s\right)^{-\gamma} \mathrm{d} s \\
& \propto \int_{0}^{1} s^{\xi^{\star}-1}(1-s)^{-\xi^{\star}} \frac{1}{s}\left(\frac{y}{s}+\beta_{1}\right)^{-\gamma} \mathrm{d} s .
\end{aligned}
$$

In particular, we readily deduce the following result :

Proposition 7. Assume $U$ is a r.v. with a scaled type of Beta law $\mathcal{B}\left(\xi^{\star}, 1-\xi^{\star}\right), U \sim c_{4} s^{\xi^{\star}-1}(1-s)^{-\xi^{\star}} \mathbb{I}_{s \in(o, 1)}$ and $M_{1}$ is a r.v. independent of $U$ with law $M_{1} \sim c_{5}\left(z+\beta_{1}\right)^{-\gamma} \mathbb{I}_{z \in(\mathrm{o}, \infty)}$. We have the identity in law

$$
Z_{1}^{0} \stackrel{\mathcal{L}}{\sim} M_{1} \times U
$$

Moreover, since $\left(Z_{t}^{0}\right)_{t \geq 0}$ is auto-similar with index 1 , we have

\section{Proposition 8.}

$$
g_{1}=\sup \left(t \leq 1: Z_{t}^{0}=0\right) \stackrel{\mathcal{L}}{\sim} 1-U
$$

Proof. Introduce $d_{u}:=\inf \left(s \geq u: Z_{s}^{0}=0\right)$. From Markov's property applied to $\left(Z_{t}^{0}\right)_{t \geq 0}$, we have for $u \leq 1$

$$
\begin{aligned}
\mathbb{P}^{0}\left(g_{1} \leq u\right) & =\mathbb{P}^{0}\left(d_{u} \geq 1\right)=\int_{0}^{\infty} p_{Z}(u, 0, y) \mathbb{P}^{y}\left(U^{*} \geq 1-u\right) \mathrm{d} y \\
& \propto \int_{0}^{\infty} \frac{\beta_{1}}{y}\left(\frac{u \beta_{1}}{y}\right)^{\gamma-1}\left(1+\frac{u \beta_{1}}{y}\right)^{1-\gamma-\xi^{\star}} \int_{1-u}^{\infty} \frac{\beta_{1}}{y}\left(\frac{t \beta_{1}}{y}\right)^{\xi^{\star}-2}\left(1-\frac{y}{\beta_{1} t}\right)^{\xi^{\star}+\gamma-3} \mathbb{I}_{t \geq \frac{y}{\beta_{1}}} \mathrm{~d} t \mathrm{~d} y \\
& \propto u^{\gamma-1} \int_{1-u}^{\infty} t^{\xi^{\star}-2-\left(\xi^{\star}+\gamma-3\right)} \int_{0}^{\beta_{1} t}\left(y+u \beta_{1}\right)^{1-\gamma-\xi^{\star}}\left(\beta_{1} t-y\right)^{\xi^{\star}+\gamma-3} \mathrm{~d} y \mathrm{~d} t \\
& \propto \int_{1-u}^{\infty}\left(\frac{u}{t}\right)^{\gamma-1} \int_{0}^{t}(y+u)^{1-\gamma-\xi^{\star}}(t-y)^{\xi^{\star}+\gamma-3} \mathrm{~d} y \mathrm{~d} t \\
& \propto \int_{1-u}^{\infty}\left(\frac{u}{t}\right)^{\gamma-1} \frac{1}{u+t} \int_{0}^{t} \frac{\partial}{\partial y}\left[-\left(\frac{y+u}{t-y}\right)^{2-\gamma-\xi^{\star}}\right] \mathrm{d} y \mathrm{~d} t \\
& \propto \int_{0}^{u /(1-u)} \tau^{-\xi^{\star}} \frac{1}{1+\tau} \mathrm{d} \tau \propto \int_{0}^{u}\left(\frac{\theta}{1-\theta}\right)^{-\xi^{\star}} \frac{1}{1+\frac{\theta}{1-\theta}} \frac{1}{(1-\theta)^{2}} \mathrm{~d} \theta \\
& \propto \int_{0}^{u} \theta^{-\xi^{\star}}(1-\theta)^{\xi^{\star}-1} \mathrm{~d} \theta .
\end{aligned}
$$

For any fixed $t>0$, set $A_{t}:=t-g_{t}$. If $F$ is a positive measurable function of the state space of excursions, set

$$
q(s, F):=\mathrm{n}(R(\mathrm{e})>s)^{-1} \int_{R>s} F(\mathrm{e}) \mathrm{n}(\mathrm{de}) .
$$

Proposition 9. (Azema's projection)

For any fixed $t>0$,

$$
\mathbb{E}^{0}\left[F\left(\mathrm{e}^{g_{t}}\right) \mid \check{\mathcal{F}}_{t}\right]=q\left(A_{t}, F\right) \quad \text { a.s. }
$$


Proof. We follow [22] Chap XII Proposition 3.3 pp 489-490 with the use of the master formula (65) stated in Appendix A.2.

We know that a.s. $t$ is not a zero of $Z$, hence $0<g_{t}<t$ and $q\left(A_{t}, F\right)$ is defined. For $s \in \bar{G} \cap[0, t]$, we have $s=g_{t}$ if and only if $s+R \circ \theta_{s}>t$ (where $\left(\theta_{s}\right)$ denotes the usual shift operator on $\Omega$ ). Consequently, if $H$ is a positive $\left(\mathcal{F}_{t}\right)$-predictable process, we have applying $(65)$ :

$$
\begin{aligned}
\mathbb{E}^{0}\left[H_{g_{t}} F\left(\mathrm{e}^{g_{t}}\right)\right] & =\mathbb{E}^{0}\left[\sum_{s \in \bar{G}} H_{s} F\left(\mathrm{e}^{s}\right) \mathbb{I}_{R \circ \theta_{s}>t-s}\right]=\mathbb{E}^{0}\left[\int_{0}^{\infty} H_{s} \mathrm{n}\left(F \mathbb{I}_{R>t-s}\right) \mathrm{d} \ell_{s}^{0}(Z)\right] \\
& =\mathbb{E}^{0}\left[\int_{0}^{\infty} H_{s} q(t-s, F) \mathrm{n}(R(\mathrm{e})>t-s) \mathrm{d} \ell_{s}^{0}(Z)\right] \\
& =\mathbb{E}^{0}\left[\sum_{s \in \bar{G}} H_{s} q(t-s, F) \mathbb{I}_{R \circ \theta_{s}>t-s}\right]=\mathbb{E}^{0}\left[H_{g_{t}} q\left(A_{t}, F\right)\right]
\end{aligned}
$$

Corollary 5. For any function $f$ such that $f\left(Z_{t}\right)$ is integrable,

$$
\mathbb{E}\left[f\left(Z_{t}\right) \mid \check{\mathcal{F}}_{t}\right]=q\left(A_{t}, f\left(\mathrm{e}^{g_{t}}\left(A_{t}\right)\right)\right) \propto\left(A_{t}\right)^{1-\xi^{\star}} \mathrm{n}_{A_{t}}(f) \propto\left(t-g_{t}\right)^{\gamma-1} \int_{0}^{\infty} f(y)\left(y+\beta_{1}\left(t-g_{t}\right)\right)^{-\gamma} \mathrm{d} y
$$

Let us now follow the lines of the construction of Azema martingales as in [22] Chap.XII Exercice 4.16 p.505. We have that for any $\left(\mathcal{F}_{t}\right)$-martingale $M_{t}$, its projection onto $\left(\check{\mathcal{F}}_{t}\right)$ is a $\left(\check{\mathcal{F}}_{t}\right)$-martingale. As an application, for $f(y)=y$, and

$$
M_{t}=Z_{t}+\beta_{1} t+\left(\frac{\kappa}{\gamma-1}-\frac{\kappa}{\gamma-2}\right) t
$$

and using formula (61) for the computation of $\mathbb{E}\left[Z_{t} \mid \check{\mathcal{F}}_{t}\right]$, we find that there exists a constant $c$ such that

$$
m_{t}:=\left(\beta_{1}+\frac{\kappa}{\gamma-1}-\frac{\kappa}{\gamma-2}\right) t+c\left(t-g_{t}\right)
$$

is an $\left(\check{\mathcal{F}}_{t}\right)$ martingale. The constant $c$ is determined by $\mathbb{E}\left(m_{t}\right)=0$.

Remark 7. (Integral representation of $p_{Z}(1,0, \mathrm{~d} y)$ )

Choose $\hat{\mathrm{n}}_{t}(\mathrm{~d} y)=t^{\frac{1-\beta_{1}}{2 \beta_{1}}}\left(y+\beta_{1} t\right)^{-\gamma} \mathrm{d} y \propto \mathrm{n}_{t}(\mathrm{~d} y)$ as the renormalization of the entrance law $\mathrm{n}_{t}(\mathrm{~d} y)$. Then,

$$
\hat{\mathrm{n}}_{t} Q_{s}(\mathrm{~d} y)=n_{t+s}(\mathrm{~d} y)=\hat{\mathrm{n}}_{t+s}(\mathrm{~d} y) \quad(s, t>0)
$$

and

$$
p_{Z}(1,0, \mathrm{~d} y)=c_{0} \int_{0}^{1} \mathrm{~d} u(1-u)^{-\xi^{\star}} \hat{\mathrm{n}}_{u}(\mathrm{~d} y)
$$

with $c_{0}=\frac{\gamma-1}{\beta_{1}^{1-\gamma}} \frac{1}{\mathcal{B}\left(\xi^{\star}, 1-\xi^{\star}\right)}$. 


\section{Markovian dependance on $\beta \in(0,1) \cap \mathbb{Q}$}

\subsection{Markov property}

For $\beta=0$ let $L_{t}^{0}:=1 \times L_{t}^{0}\left(X^{0,0}\right)=L_{t}^{0}(B)$ and for $\beta \in(0,1)$ let $L_{t}^{\beta}:=\beta L_{t}^{0}\left(X^{0, \beta}\right)$, where $L_{t}^{0}\left(X^{0, \beta}\right)$ is the local time process corresponding to $\left(X_{t}^{\beta}\right)$ with parameter $\beta$ and starting from $x=0$. Unique strong solutions exist for all rational $\beta \in[0,1)$ simultaneously.

Fix some rational $\beta_{2} \in(0,1) \in \mathbb{Q}$ and let $\beta_{1}<\beta_{2}<\beta_{3}$. The process $\left(X_{t}^{\beta_{2}}\right)$ is a Skew Brownian Motion. Let $\mathcal{E}^{+}=\left\{\left(s, \mathbf{e}_{s}\right)^{+}\right\}_{s \in G_{+}^{\beta_{2}}}$ be the Poisson point process of positive excursions of $\left(X_{t}^{\beta_{2}}\right)$. Here $G_{+}^{\beta_{2}}$ is the set of all $s \geq 0$ such that for some $0<g_{s}<d_{s}$, we have $s=L_{g_{s}}^{0}\left(X^{0, \beta_{2}}\right), X_{g_{s}}^{0, \beta_{2}}=0=X_{d_{s}}^{0, \beta_{2}}, X_{v}^{0, \beta_{2}}>0$ for $v \in\left(g_{s}, d_{s}\right)$ and $\mathbf{e}_{s}^{+}(u)=X_{g_{s}+u}^{0, \beta_{2}}$ for $u \in\left[0, d_{s}-g_{s}\right)$.

We define in analogous way the Poisson point process $\mathcal{E}^{-}=\left\{\left(s, \mathbf{e}_{s}\right)^{-}\right\}_{s \in G_{-}^{\beta_{2}}}$ of negative excursions of $\left(X_{t}^{\beta_{2}}\right)$. The processes $\mathcal{E}^{+}$and $\mathcal{E}^{-}$are independent.

Let $\mathcal{L}_{s}^{\beta_{3}}=\mathcal{L}_{s}^{\beta_{3}, \beta_{2}}:=\inf \left\{L_{t}^{\beta_{3}}: L_{t}^{\beta_{2}}>s\right\}=L_{\tau_{s / \beta_{2}}^{\beta_{2}}}^{\beta_{3}}$. Define $\mathcal{L}_{s}^{\beta_{1}}$ and $\mathcal{L}_{s}^{0}$ in a similar way.

We have the inequalities $L_{t}^{\beta_{2}} \geq L_{t}^{\beta_{1}}$ for all $t \geq 0$ a.s. On the intervals where $\left(X_{t}^{\beta_{2}}\right)$ is strictly negative the value of the processes $L_{t}^{\beta_{2}}, L_{t}^{\beta_{1}}$ and $L_{t}^{0}$ do not change. In turn this implies that $\mathcal{L}_{s}^{\beta_{1}}, \mathcal{L}_{s}^{0}$ are measurable with respect to the filtration $\mathcal{F}^{+}=\sigma\left\{\left(s, \mathbf{e}_{s}\right)^{+}\right\}_{s \in G_{+}^{\beta_{2}}, s \leq u}$. The same reasoning ensures that $\mathcal{L}_{s}^{\beta_{3}}$ is adapted to $\sigma\left\{\left(s, \mathbf{e}_{s}\right)^{-}\right\}_{s \in G_{+}^{\beta_{2}}, s \leq u}$. The random time $\mathcal{T}=\inf \left(s: \mathcal{L}_{s}^{0} \geq 1\right)=L_{\tau_{1}^{0}}^{\beta_{2}}$ is a stopping time relative to $\mathcal{F}^{+}$. By independence of $\mathcal{E}^{+}$and $\mathcal{E}^{-}$, the random elements $\mathcal{L}_{\mathcal{T}}^{\beta_{1}}$ and $\mathbf{e}_{\mathcal{T}}^{+}$are independent of $\mathcal{L}_{\mathcal{T}}^{\beta_{3}}$ given the value of $\mathcal{T}=L_{\tau_{1}^{0}}^{\beta_{2}}$. But this can be restated as the independence of $L_{\tau_{1}^{0}}^{\beta_{1}}$ and $L_{\tau_{1}^{0}}^{\beta_{3}}$ given $L_{\tau_{1}^{0}}^{\beta_{2}}$, since $\mathcal{L}_{\mathcal{T}}^{\beta_{3}}=L_{\tau_{1}^{0}}^{\beta_{3}}$ and $L_{\tau_{1}^{0}}^{\beta_{1}}$ is a function of $\mathcal{L}_{\mathcal{T}}^{\beta_{1}}$ and $\mathbf{e}_{\mathcal{T}}^{+}$. This proves the Markov property for the process $\beta \in[0,1) \cap \mathbb{Q} \mapsto L_{\tau_{1}^{0}}^{\beta}$.

\subsection{The description of the process $\beta \in(0,1) \cap \mathbb{Q} \mapsto X_{\tau_{1}^{0}}^{\beta}$}

Conditionally on $L_{\tau_{1}^{0}}^{\beta}=a$, we have that

$$
X_{\tau_{1}^{0}}^{\beta+\varepsilon}-X_{\tau_{1}^{0}}^{\beta}=X_{\tau_{\frac{a}{\beta}}^{\beta}}^{\beta+\varepsilon}-X_{\tau_{\frac{a}{\beta}}^{\beta}}^{\beta}
$$

In particular, conditionally on $L_{\tau_{1}^{0}}^{\beta}=a$

$$
X_{\tau_{1}^{0}}^{\beta+\varepsilon}=Z_{\frac{a}{\beta}}^{\beta+\varepsilon}+a
$$

In particular for $\beta+\varepsilon>\beta>\frac{\beta+\varepsilon}{1+2(\beta+\varepsilon)}>0$ i.e. for $\frac{2 \beta^{2}}{1-\beta}>\varepsilon>0$

$$
X_{\tau_{1}^{0}}^{\beta+\varepsilon}=Z_{\frac{a}{\beta}}^{\beta+\varepsilon}+a .
$$


Hence, we find that for any $\varepsilon \in \mathbb{Q}$ such that $\frac{2 \beta^{2}}{1-\beta}>\varepsilon>0$,

$$
\begin{aligned}
\mathcal{L}\left(X_{\tau_{1}^{0}}^{\beta+\varepsilon} \mid X_{\tau_{1}^{0}}^{\beta}=a\right) & =c_{1}(\beta, \beta+\varepsilon) \frac{1}{y-a}\left(\frac{a}{y-a}\right)^{\frac{1+(\beta+\varepsilon)}{2(\beta+\varepsilon)}}\left(1+\frac{a}{y-a}\right)^{-\frac{1+\beta}{2 \beta}} \mathbb{I}_{y>a} \mathrm{~d} y \\
& =c_{1}(\beta, \beta+\varepsilon) \frac{1}{y-a}\left(\frac{a}{y-a}\right)^{\frac{1+(\beta+\varepsilon)}{2(\beta+\varepsilon)}}\left(\frac{y}{y-a}\right)^{-\frac{1+\beta}{2 \beta}} \mathbb{I}_{y>a} \mathrm{~d} y \\
& :=q_{\beta, \beta+\varepsilon}(a, y) \mathrm{d} y .
\end{aligned}
$$

The process $\beta \in(0,1) \cap \mathbb{Q} \mapsto X_{\tau_{1}^{0}}^{\beta}$ is an a.s. increasing process.

Let us check the Chapman-Kolmogorov equations. Indeed, for any $y>a, \varepsilon \in \mathbb{Q}$ such that $\frac{2 \beta^{2}}{1-\beta}>\varepsilon>0$, and $\theta \in \mathbb{Q}$ such that $\frac{2(\beta+\varepsilon)^{2}}{1-(\beta+\varepsilon)}>\theta>0$ :

$$
\begin{aligned}
& \int q_{\beta, \beta+\varepsilon}(a, z) q_{\beta+\varepsilon, \beta+\varepsilon+\theta}(z, y) \mathrm{d} z \\
& \propto \int_{a}^{y} \frac{1}{z-a}\left(\frac{a}{z-a}\right)^{\frac{1+(\beta+\varepsilon)}{2(\beta+\varepsilon)}}\left(\frac{z}{z-a}\right)^{-\frac{1+\beta}{2 \beta}} \frac{1}{y-z}\left(\frac{z}{y-z}\right)^{\frac{1+(\beta+\varepsilon+\theta)}{2(\beta+\varepsilon+\theta)}}\left(\frac{y}{y-z}\right)^{-\frac{1+(\beta+\varepsilon)}{2(\beta+\varepsilon)}} \mathrm{d} z \\
& \propto \int_{a}^{y} \frac{1}{z-a}\left(\frac{a}{y} \frac{y-z}{z-a}\right)^{\frac{1+(\beta+\varepsilon)}{2(\beta+\varepsilon)}}\left(\frac{z}{z-a}\right)^{-\frac{1+\beta}{2 \beta}} \frac{1}{y-z}\left(\frac{z}{y-z}\right)^{\frac{1+(\beta+\varepsilon+\theta)}{2(\beta+\varepsilon+\theta)}} \mathrm{d} z,
\end{aligned}
$$

and performing the change of variable $\zeta=\frac{y-z}{z-a}$,

$$
\begin{aligned}
& \int q_{\beta, \beta+\varepsilon}(a, z) q_{\beta+\varepsilon, \beta+\varepsilon+\theta}(z, y) \mathrm{d} z \\
& \propto\left(\frac{a}{y}\right)^{\frac{1+(\beta+\varepsilon)}{2(\beta+\varepsilon)}} \frac{1}{y-a} \int_{0}^{\infty} \zeta^{\frac{1+(\beta+\varepsilon)}{2(\beta+\varepsilon)}}\left(\frac{y+a \zeta}{y-a}\right)^{-\frac{1+\beta}{2 \beta}} \frac{1}{\zeta}\left(\frac{y+a \zeta}{(y-a) \zeta}\right)^{\frac{1+(\beta+\varepsilon+\theta)}{2(\beta+\varepsilon+\theta)}} \mathrm{d} \zeta \\
& \propto\left(\frac{a}{y}\right)^{\frac{1+(\beta+\varepsilon)}{2(\beta+\varepsilon)}}\left(\frac{1}{y-a}\right)^{1-\frac{1+\beta}{2 \beta}}\left(\frac{1}{y-a}\right)^{\frac{1+(\beta+\varepsilon+\theta)}{2(\beta+\varepsilon+\theta)}} \\
& \quad \times \int_{0}^{\infty} \zeta^{\frac{1+(\beta+\varepsilon)}{2(\beta+\varepsilon)}-1-\frac{1+(\beta+\varepsilon+\theta)}{2(\beta+\varepsilon+\theta)}}(y+a \zeta)^{-\frac{1+\beta}{2 \beta}+\frac{1+(\beta+\varepsilon+\theta)}{2(\beta+\varepsilon+\theta)}} \mathrm{d} \zeta,
\end{aligned}
$$

so that

$$
\begin{aligned}
& \int q_{\beta, \beta+\varepsilon}(a, z) q_{\beta+\varepsilon, \beta+\varepsilon+\theta}(z, y) \mathrm{d} z \\
& \propto y^{-\frac{1+\beta}{2 \beta}+\frac{1+(\beta+\varepsilon+\theta)}{2(\beta+\varepsilon+\theta)}}\left(\frac{a}{y}\right)^{\frac{1+(\beta+\varepsilon)}{2(\beta+\varepsilon)}}\left(\frac{a}{y}\right)^{-\frac{1+(\beta+\varepsilon)}{2(\beta+\varepsilon)}+1+\frac{1+(\beta+\varepsilon+\theta)}{2(\beta+\varepsilon+\theta)}}\left(\frac{1}{y-a}\right)^{1-\frac{1+\beta}{2 \beta}}\left(\frac{1}{y-a}\right)^{\frac{1+(\beta+\varepsilon+\theta)}{2(\beta+\varepsilon+\theta)}} \\
& \quad \times \int_{0}^{\infty}\left(\frac{a}{y} \zeta\right)^{\frac{1+(\beta+\varepsilon)}{2(\beta+\varepsilon)}-1-\frac{1+(\beta+\varepsilon+\theta)}{2(\beta+\varepsilon+\theta)}}\left(1+\frac{a}{y} \zeta\right)^{-\frac{1+\beta}{2 \beta}+\frac{1+(\beta+\varepsilon+\theta)}{2(\beta+\varepsilon+\theta)}} \mathrm{d} \zeta .
\end{aligned}
$$

Finally,

$$
\begin{aligned}
& \int q_{\beta, \beta+\varepsilon}(a, z) q_{\beta+\varepsilon, \beta+\varepsilon+\theta}(z, y) \mathrm{d} z \\
& \propto y^{-\frac{1+\beta}{2 \beta}+\frac{1+(\beta+\varepsilon+\theta)}{2(\beta+\varepsilon+\theta)}}\left(\frac{a}{y}\right)^{\frac{1+(\beta+\varepsilon+\theta)}{2(\beta+\varepsilon+\theta)}}\left(\frac{1}{y-a}\right)^{1-\frac{1+\beta}{2 \beta}}\left(\frac{1}{y-a}\right)^{\frac{1+(\beta+\varepsilon+\theta)}{2(\beta+\varepsilon+\theta)}} \mathbb{I}_{y>a} \\
& \propto\left(\frac{1}{y-a}\right)\left(\frac{y}{y-a}\right)^{-\frac{1+\beta}{2 \beta}}\left(\frac{a}{y-a}\right)^{\frac{1+(\beta+\varepsilon+\theta)}{2(\beta+\varepsilon+\theta)}} \mathbb{I}_{y>a} \\
& \propto q_{\beta, \beta+\varepsilon+\theta}(a, y) .
\end{aligned}
$$


Let us now compute the infinitesimal generators $\left(\mathfrak{G}_{\beta}\right)_{\beta \in(0,1) \cap \mathbb{Q}}$ of the inhomogeneous Markov process $\beta \in$ $(0,1) \cap \mathbb{Q} \mapsto X_{\tau_{1}^{0}}^{\beta}$. Using $\frac{1}{\Gamma(z)} \sim_{0} z \mathrm{e}^{\rho z}$ ( $\rho$ is the Euler constant $)$ :

$$
\begin{aligned}
\mathfrak{G}_{\beta} g(a) & =\lim _{\varepsilon \searrow 0+} \frac{1}{\varepsilon} \int_{0}^{\infty}(g(a+z)-g(a)) \frac{1}{\Gamma\left(\frac{1}{2 \beta}-\frac{1}{2(\beta+\varepsilon)}\right)} \frac{1}{z}\left(\frac{a}{z}\right)^{\frac{1+(\beta+\varepsilon)}{2(\beta+\varepsilon)}}\left(\frac{z+a}{z}\right)^{-\frac{1+\beta}{2 \beta}} \mathrm{d} z \\
& =\int_{0}^{\infty}(g(a+z)-g(a)) \frac{1}{2 \beta^{2}} \frac{1}{z}\left(\frac{a}{a+z}\right)^{\frac{1+\beta}{2 \beta}} \mathrm{d} z .
\end{aligned}
$$

Note that the ladder integral is not well definite in general if $g$ is only assumed to be bounded in some neighborhood of $a$, so it is not clear to answer what is the domain of $\mathfrak{G}_{\beta}$. If we assume that $g$ is bounded and is uniformly a Hölder function in the sense that there exists $\eta>0$ and $\delta>0$ such that

$$
\sup _{a \in \mathbb{R}^{+}} \sup _{z \in[0, \delta[} \frac{g(a+z)-g(a)}{z^{\eta}}<\infty,
$$

then $\mathfrak{G}_{\beta} g$ is well-defined of all $\beta \in(0,1) \cap \mathbb{Q}$.

\section{A Appendix : brief recalls about extensions of self-similar Markov processes}

\section{A.1 Positive self-similar Markov processes}

In his pioneering study [17] of the structure of self-similar Markov processes with state space [0, $\infty)$ Lamperti posed the problem of determining those self-similar Markov processes that agree with a given self-similar Markov process up to the time the latter process first hits 0. A few years ago, Vuolle-Apiala [24], Rivero [20], [21], and Fitzsimmons [12], pushed forward the studies of this problem by giving necessary and sufficient condition for the existence of such a "recurrent extension" that, in addition, leaves 0 continuously. To state the main results of this beautiful theory precisely, we introduce some notation and recall some of the basic theory of self-similar Markov processes. A Borel right process $Z^{\dagger}=\left(\left(Z_{t}^{\dagger}\right)_{t \geq 0},\left(\mathbb{P}^{x}\right)_{x \geq 0}\right)$ with values in $[0, \infty)$ is self-similar provided there exists $\alpha>0$ such that, for each $c>0$ and $x \geq 0$, the law of the rescaled process $\left(c^{-\alpha} Z_{c t}\right)_{t \geq 0}$, is $\mathbb{P}^{x / c}$ when $Z^{\dagger}$ has law $\mathbb{P}^{x}$. The number $\alpha$ is the order of $Z^{\dagger}$. Thanks to [17], we can assume that $Z$ is a Hunt process; thus in addition to being a right-continuous strong Markov process, the sample paths of $Z$ are quasi-left-continuous. One of several zero-one laws developed by Lamperti states that if $T_{0}:=\inf \left\{t>0: Z_{t}^{\dagger}=0\right\}$ then either $\mathbb{P}^{x}\left[T_{0}<\infty\right]=0$ for all $x>0$ or $\mathbb{P}^{x}\left[T_{0}<\infty\right]=1$ for all $x>0$. Let us assume the ladder in this section.

We assume that 0 is a trap for $Z^{\dagger}$, so that each of the laws $\mathbb{P}^{x}$ governing $Z^{\dagger}$ is carried by $\left\{\omega \in \Omega: \omega(t)=0, \forall t \geq T_{0}(\omega)\right\}$. The natural filtration on $\Omega$ is $\left(\mathcal{G}_{t}\right)_{t \geq 0}$, and $\mathcal{G}_{\infty}:=\bigvee_{t \geq 0} \mathcal{G}_{t}$. We write

$$
P_{t} f(x)=P_{t}(x, f):=\mathbb{P}^{x}\left[f\left(Z_{t}^{\dagger}\right)\right]
$$

for the transition semigroup of $Z^{\dagger}$, and

$$
U^{q}:=\int_{0}^{\infty} \mathrm{e}^{-q t} P_{t} \mathrm{~d} t
$$


$(q>0)$, for the associated resolvent operators.

Define, for $c>0, \phi_{c}: \Omega \rightarrow \Omega$ by $\phi_{c} \omega(t):=c^{-\alpha} \omega(c t)$. The $\alpha$-self-similarity of $Z^{\dagger}$ means that

$$
\phi_{c} \mathbb{P}^{x}(B):=\mathbb{P}^{x}\left[\phi_{c}^{-1} B\right]=\mathbb{P}^{x / c^{\alpha}}(B) \quad \forall B \in \mathcal{G}_{\infty}, x \geq 0, c>0 .
$$

Definition 2. A Borel right Markov process $Z=\left(Z_{t}, \mathbb{P}^{x}\right)$ with state space $[0, \infty)$ is a recurrent extension of $Z^{\dagger}$ provided :

(i) the stopped process $\left(\left(Z_{t \wedge T_{0}}\right)_{t \geq 0}, \mathbb{P}^{x}\right)$ has the same law as $\left(\left(Z_{t}^{\dagger}\right)_{t \geq 0}, \mathbb{P}^{x}\right)$, for each $x \geq 0$

(ii) 0 is not a trap for $Z$.

Note that if a recurrent extension $Z$ is self-similar, then its order must be the same as that of $Z^{\dagger}$.

Let $Z$ a self-similar recurrent extension of $Z^{\dagger}$. Then its order must be same as that of $Z^{\dagger}$.

\section{A.2 Excursions associated to markovian extensions}

Let $\bar{M}$ denote the closure of the zero set $\left\{t \geq 0: Z_{t}=0\right\}$ and let $\bar{G}$ denote the set of strictly positive left endpoints of the maximal open sets in the complement of $\bar{M}$. The excursions of $Z$ from 0 are indexed by elements of $\bar{G}$. The excursion $\mathrm{e}^{s}$ associated with $s \in \bar{G}$ is the $\mathcal{D}$ valued path defined by

$$
\mathrm{e}^{s}(t):=\left\{\begin{array}{l}
Z_{t} \circ \theta_{s}=Z_{t+s}, \quad 0 \leq t<T_{0} \circ \theta_{s}, \\
0, \quad t \geq T_{0} \circ \theta_{s}
\end{array}\right.
$$

where $\theta_{s}$ is the shift operator on $\mathcal{D}$. Let $\left(\ell_{t}^{0}(Z)\right)_{t \geq 0}$ denote the $Z$-local time at 0 , normalized so that $\mathbb{P}^{0} \int_{0}^{\infty} \mathrm{e}^{-t} \mathrm{~d} \ell_{t}^{0}(Z)=$ 1.

Then, there is a $\sigma$-finite measure $\mathrm{n}$ on $\left(\Omega, \mathcal{G}_{\infty}\right)$ such that for any predictable $V \geq 0$ and $\mathcal{G}_{\infty}$-measurable $F \geq 0$,

$$
\mathbb{E}^{0}\left[\sum_{s \in \bar{G}} V_{s} \cdot F\left(\mathrm{e}^{s}\right)\right]=\mathbb{E}^{0}\left[\int_{0}^{\infty} V_{s} \mathrm{~d} \ell_{s}^{0}(Z)\right] \cdot \mathrm{n}(F) .
$$

This formula determines $\mathrm{n}$ uniquely, and under $\mathrm{n}$, the process $\left(Z_{t}\right)_{t \geq 0}$ is a strong Markov process.

We will use a slight extension of (64), namely

$$
\mathbb{E}^{0}\left[\sum_{s \in \bar{G}} V_{s} \cdot F\left(s, \mathrm{e}^{s}\right)\right]=\mathbb{E}^{0}\left[\int_{0}^{\infty} V_{s} \mathrm{n}(F(s, .)) \mathrm{d} \ell_{s}^{0}(Z)\right]
$$

holding for any measurable $F:\left(\mathbb{R} \times \mathcal{C}_{0 \rightarrow 0}, \mathcal{B}(\mathbb{R}) \otimes \mathcal{U}\right) \rightarrow \mathbb{R}$ (where $\mathcal{U}$ stands for the Borel $\sigma$-algebra of $\mathcal{C}_{0 \rightarrow 0}$ ).

It is shown in [24] (see Theorem 1.2) that if $Z$ is a self-similar recurrent extension of $Z^{\dagger}$ then

$$
\int_{0}^{\infty} \mathbb{I}_{\{0\}}\left(Z_{s}\right) \mathrm{d} s=0
$$

and

$$
\text { either } \mathrm{n}\left(Z_{0}>0\right)=0 \quad \text { or } \quad \mathrm{n}\left(Z_{0}=0\right)=0 \text {. }
$$

Definition 3. We say that the extension $\left(Z_{t}\right)_{t \geq 0}$ leaves 0 continuously if

$$
\mathrm{n}\left(Z_{0}>0\right)=0 .
$$




\section{A.3 The underlying Lévy process and Cramer's condition}

Some of our results will be stated in terms of the Lévy process associated with $Z^{\dagger}$ by the Lamperti transformation. For this, consider the continuous additive functional $A$ defined by

$$
A_{t}=\int_{0}^{t}\left(Z_{s}^{\dagger}\right)^{-\alpha} \mathrm{d} s, \quad t \geq 0,
$$

and its right continuous inverse $\tau$ defined by

$$
\tau_{u}:=\inf \left\{t>0: A_{t}>u\right\}, \quad u \geq 0
$$

in which we follow the usual convention inf $\emptyset=+\infty$. Then, according to [17] the $[-\infty,+\infty]$-valued process

$$
H_{u}:=\ln \left(Z_{\tau_{u}}^{\dagger}\right), \quad u \geq 0
$$

is Lévy process. This process is referred to as the Lévy process underlying $Z^{\dagger}$.

We have the following Theorem

Theorem 7. 1. The $\alpha$-self similar Markov process $Z^{\dagger}$ admits a self-similar recurrent extension that leaves 0 continuously if and only if there exists $\theta \in\left(0, \alpha^{-1}\right)$ such that condition

$$
\mathbb{E}^{0}\left(\exp \left(\theta H_{1}\right)\right)=1
$$

holds.

2. There is at most one self-similar recurrent extension that leaves 0 continuously.

\section{References}

[1] Milton Abramowitz and Irene A. Stegun. Handbook of mathematical functions with formulas, graphs, and mathematical tables, volume 55 of National Bureau of Standards Applied Mathematics Series. For sale by the Superintendent of Documents, U.S. Government Printing Office, Washington, D.C., tenth printing edition, 1972.

[2] Martin Barlow, Krzysztof Burdzy, Haya Kaspi, and Avi Mandelbaum. Coalescence of skew Brownian motions. In Séminaire de Probabilités, XXXV, volume 1755 of Lecture Notes in Math., pages $202-205$. Springer, Berlin, 2001.

[3] Richard F. Bass and Zhen-Qing Chen. One-dimensional stochastic differential equations with singular and degenerate coefficients. Sankhyā, 67(1):19-45, 2005.

[4] Jean Bertoin. Subordinators : examples and applications. In Ecole d'été de Probabilité de St-Flour, 1997.

[5] Krzysztof Burdzy. Differentiability of stochastic flow of reflected Brownian motions. Electron. J. Probab., 14:no. 75, 2182-2240, 2009. 
[6] Krzysztof Burdzy and Zhen-Qing Chen. Local time flow related to skew Brownian motion. Ann. Probab., 29(4):1693-1715, 2001.

[7] Krzysztof Burdzy, Zhen-Qing Chen, and Peter Jones. Synchronous couplings of reflected Brownian motions in smooth domains. Illinois J. Math., 50(1-4):189-268 (electronic), 2006.

[8] Krzysztof Burdzy and Haya Kaspi. Lenses in skew Brownian flow. The Annals of Probability 2004, Vol. 32, No. 4, 3085?3115, Institute of Mathematical Statistics, 2004

[9] Yu-Ting Chen, Cheng-Few Lee, and Yuan-Chung Sheu. An ODE approach for the expected discounted penalty at ruin in a jump-diffusion model. Finance Stoch., 11(3):323-355, 2007.

[10] M. Cranston and Y. Le Jan. On the noncoalescence of a two point Brownian motion reflecting on a circle. Ann. Inst. H. Poincaré Probab. Statist., 25(2):99-107, 1989.

[11] Robert J. Elliott. A continuous time Kronecker's lemma and martingale convergence. Stochastic Anal. Appl., 19(3):433-437, 2001.

[12] P.J. Fitzsimmons. On the existence of reccurent extensions of self-similar Markov processes Elect. Comm. in Probab. 11 (2006), 230-241.

[13] A. Gloter and M. Martinez. Distance between two skew Brownian motions as a S.D.E. with jumps and law of the hitting time. Anal. Of. Prob., 2012.

[14] F.M. Guillemin and R.R. Mazumbar. Forward Equations for Reflected Diffusions with Jumps. Appl. Math. Optim., 33:81-102, 1996.

[15] J. M. Harrison and L. A. Shepp. On skew Brownian motion. Ann. Probab., 9(2):309-313, 1981.

[16] Jean Jacod and Albert N. Shiryaev. Limit theorems for stochastic processes, volume 288 of Grundlehren der Mathematischen Wissenschaften [Fundamental Principles of Mathematical Sciences]. Springer-Verlag, Berlin, 1987.

[17] J. Lamperti. Semi-stable Markov processes I. z. Wahrscheinlichkeitstheorieverw. Geb. 205-225 (1972).

[18] A. Lejay. On the constructions of the skew Brownian motion. Probab. Surv., 3:413-466 (electronic), 2006.

[19] J. Pitman and M. Yor. The two-parameter Poisson-Dirichlet distribution derived from a stable subordinator Ann. Probab., Vol. 25, No. 2, 855-900, 1997.

[20] V. Rivero. Recurrent extensions of self-similar Markov processes and Cramer's condition Bernoulli 11(3), 471-509, 2005.

[21] V. Rivero. Recurrent extensions of self-similar Markov processes and Cramer's condition II Bernoulli 13(4), 1053-1070, 2007. 
[22] Daniel Revuz and Marc Yor. Continuous martingales and Brownian motion, volume 293 of Grundlehren der Mathematischen Wissenschaften [Fundamental Principles of Mathematical Sciences]. Springer-Verlag, Berlin, third edition, 1999.

[23] S.G. Samko, A.A. Kilbas, O.I. Marichev. Fractional integrals and derivatives Theory and applications Gordon and Breach Science Publishers S.A. 1987, Nauka i Technika, Minsk

[24] J. Vuolle-Apalia. Itô excursion theory for self-similar Markov processes. Ann. Probab., 22(2), p. 546-565. 